\title{
Construction and nonexistence of strong external difference families
}

\author{
Jonathan Jedwab ${ }^{1}$ (D) Shuxing Li ${ }^{1}$
}

Received: 28 January 2017 / Accepted: 23 January 2018 / Published online: 1 February 2018

C Springer Science+Business Media, LLC, part of Springer Nature 2018

\begin{abstract}
Strong external difference families (SEDFs) were introduced by Paterson and Stinson as a more restrictive version of external difference families. SEDFs can be used to produce optimal strong algebraic manipulation detection codes. We characterize the parameters $(v, m, k, \lambda)$ of a nontrivial SEDF that is near-complete (satisfying $v=k m+1)$. We construct the first known nontrivial example of a $(v, m, k, \lambda)$ SEDF having $m>2$. The parameters of this example are $(243,11,22,20)$, giving a nearcomplete SEDF, and its group is $\mathbb{Z}_{3}^{5}$. We provide a comprehensive framework for the study of SEDFs using character theory and algebraic number theory, showing that the cases $m=2$ and $m>2$ are fundamentally different. We prove a range of nonexistence results, greatly narrowing the scope of possible parameters of SEDFs.
\end{abstract}

Keywords Character sum · Exponent bound · Finite projective geometry · Mathieu group $\cdot$ Partial difference set $\cdot$ Strong external difference family

\section{Introduction}

Let $G$ be an abelian group of order $v$ with identity 1 . We shall work in the setting of the group ring $\mathbb{Z}[G]$ : given a subset $D$ of $G$, we write the group ring element $\sum_{d \in D} d$ as

\section{J. Jedwab is supported by NSERC.}

Jonathan Jedwab

jed@sfu.ca

Shuxing Li

shuxing_li@sfu.ca

1 Department of Mathematics, Simon Fraser University, 8888 University Drive, Burnaby, BC V5A 1S6, Canada 
$D$ (by a standard abuse of notation), and the group ring element $\sum_{d \in D} d^{-1}$ as $D^{(-1)}$. Let $D_{1}, D_{2}, \ldots, D_{m}$ be mutually disjoint $k$-subsets of $G$, where $m \geq 2$, and let $\lambda$ be a positive integer. Then $\left\{D_{1}, D_{2}, \ldots, D_{m}\right\}$ is a $(v, m, k, \lambda)$-external difference family in $G$ if

$$
\sum_{\substack{1 \leq i, j \leq m \\ i \neq j}} D_{j} D_{i}^{(-1)}=\lambda(G-1) \text { in } \mathbb{Z}[G],
$$

and is a $(v, m, k, \lambda)$-strong external difference family (SEDF) in $G$ if

$$
D_{j} \sum_{\substack{1 \leq i \leq m \\ i \neq j}} D_{i}^{(-1)}=\lambda(G-1) \text { in } \mathbb{Z}[G] \text { for each } j \text { satisfying } 1 \leq j \leq m
$$

The use of "strong" arises because a $(v, m, k, \lambda)$-SEDF is necessarily a $(v, m, k, m \lambda)$ external difference family.

External difference families have applications in authentication codes and secret sharing [23]. An external difference family in a cyclic group gives rise to difference systems of sets [7], which can be applied to construct synchronization codes [18]. Paterson and Stinson [25] introduced SEDFs and showed how to produce optimal strong algebraic manipulation detection codes from them. Algebraic manipulation detection codes have many applications, including robust secret sharing schemes, secure multiparty computation, and nonmalleable codes [8-10]. A succession of recent papers has demonstrated that SEDFs are interesting combinatorial objects in their own right: see Proposition 1.1 for a summary of constructive results, Proposition 1.3 for a characterization result, and Proposition 1.4 for a selection of nonexistence results.

The parameters of a $(v, m, k, \lambda)$-SEDF satisfy the counting relation

$$
k^{2}(m-1)=\lambda(v-1) .
$$

A ( $v, m, k, \lambda)$-SEDF is trivial if $k=1$; it follows from (1.3) that the parameters of a trivial SEDF have the form $(v, v, 1,1)$, and an SEDF with these parameters exists (trivially) in every group of order $v$. The following proposition describes the parameters and groups of the known nontrivial SEDFs, all of which satisfy $m=2$.

Proposition 1.1 $A(v, m, k, \lambda)-S E D F$ exists in the group $G$ in each of the following cases:

(1) $(v, m, k, \lambda)=\left(k^{2}+1,2, k, 1\right)$ and $G=\mathbb{Z}_{k^{2}+1}$ [25, Example 2.2].

(2) $(v, m, k, \lambda)=\left(v, 2, \frac{v-1}{2}, \frac{v-1}{4}\right)$ and $v \equiv 1(\bmod 4)$, provided there exists $a$ ( $v, \frac{v-1}{2}, \frac{v-5}{4}, \frac{v-1}{4}$ ) partial difference set in $G$ [11, Section 3], [15, Theorem 4.4].

(3) $(v, m, k, \lambda)=\left(p, 2, \frac{p-1}{4}, \frac{p-1}{16}\right)$ where $p=16 t^{2}+1$ is a prime and $t$ is an integer, and $G=\mathbb{Z}_{p}[3$, Theorem 4.3].

(4) $(v, m, k, \lambda)=\left(p, 2, \frac{p-1}{6}, \frac{p-1}{36}\right)$ where $p=108 t^{2}+1$ is a prime and $t$ is an integer, and $G=\mathbb{Z}_{p}[3$, Theorem 4.6].

Remark 1.2 Proposition 1.1 (3) describes a construction which was presented in [3, Theorem 4.3] with a prime power $q$ in place of the prime $p$ and with $G=\mathbb{F}_{q}$ in place 
of $G=\mathbb{Z}_{p}$. However, if $q=16 t^{2}+1$ is a prime power and $t$ is an integer, then $q$ must be a prime because Catalan's conjecture is known to hold [22].

When $\lambda=1$, the parameters of a nontrivial $(v, m, k, \lambda)$-SEDF have been characterized.

Proposition 1.3 A nontrivial $(v, m, k, 1)-S E D F$ exists if and only if $m=2$ and $v=$ $k^{2}+1$ [25, Theorem 2.3].

The following proposition describes parameter sets $(v, m, k, \lambda)$ for which a nontrivial SEDF is known not to exist in all groups of order $v$.

Proposition 1.4 A nontrivial ( $v, m, k, \lambda)$-SEDF does not exist in each of the following cases:

(1) $m \in\{3,4\}[21$, Theorems 3.3 and 3.6]

(2) $m>2$ and $v$ is prime [21, Theorem 3.9]

(3) $m>2$ and $\lambda=2$ [15, Corollary 3.2]

(4) $m>2$ and $\lambda>1$ and $\frac{\lambda(k-1)(m-2)}{(\lambda-1) k(m-1)}>1$ [15, Theorem 3.5]

(5) $m>2$ and there is a prime $p$ dividing $v$ for which $\operatorname{gcd}(\mathrm{km}, \mathrm{p})=1$ and $m \not \equiv 2$ $(\bmod p)$ [3, Theorem 3.5].

(6) $\lambda \geq k[3$, Lemma 1.1]

It is known [21, Lemma 1.2] that if $v=k m$, then an $(v, m, k, \lambda)$-SEDF is necessarily trivial. The same proof idea as in [21] gives the following generalization.

Lemma 1.5 Suppose there exists a $(v, m, k, \lambda)-S E D F$ for which $\operatorname{gcd}(k, v-1)=1$. Then the SEDF is trivial.

Proof The counting relation (1.3) gives $m-1=\frac{\lambda}{k^{2}}(v-1)$. Since $\operatorname{gcd}(k, v-1)=1$, it follows that $\lambda / k^{2}$ is an integer and so $m-1 \geq v-1$. Since $v \geq k m$, this implies that $k=1$.

Lemma 1.5 implies that the parameters of a nontrivial $(v, m, k, \lambda)-\operatorname{SEDF}\left\{D_{1}, D_{2}\right.$, $\left.\ldots, D_{m}\right\}$ satisfy $v>k m$ and, by taking a translate of all the subsets $D_{j}$ if necessary, we may therefore assume that $1 \notin \bigcup_{j=1}^{m} D_{j}$. In the extremal case $v=k m+1$, the subsets $D_{1}, D_{2}, \ldots, D_{m}$ partition the nonidentity elements of the group $G$ and (following [11]) we call the SEDF near-complete.

In this paper, we present constructive and nonexistence results for nontrivial SEDFs using character theory and algebraic number theory. In Sect. 2, we give a charactertheoretic framework for the study of SEDFs and demonstrate that the cases $m=2$ and $m>2$ are fundamentally different. In Sect. 3, we characterize the parameters of a nontrivial near-complete SEDF by establishing an equivalence with a collection of partial difference sets. In particular, we construct a near-complete $(243,11,22,20)$ SEDF in $\mathbb{Z}_{3}^{5}$ by reference to the point-orbits of the Mathieu group $M_{11}$ acting on the projective geometry $P G(4,3)$. This is the first known nontrivial example of an SEDF with $m>2$. In Sect. 4, we use algebraic number theory to obtain an exponent bound on a group containing a SEDF and apply it to rule out various SEDFs with $m=2$, 
leaving only 5 open cases for the parameters of a $(v, m, k, \lambda)$ SEDF with $v \leq 50$ and $m=2$. In Sect. 5 we obtain nonexistence results for SEDFs with $m>2$, introducing the "simple character value property" under which strong necessary conditions can be derived. This leaves only 70 open cases for the parameters of a $(v, m, k, \lambda)$ SEDF with $v \leq 10^{4}$ and $m>2$.

\section{A character-theoretic approach}

Let $\widehat{G}$ denote the character group of an abelian group $G$, and let $\chi_{0} \in \widehat{G}$ be the principal character. Each character $\chi \in \widehat{G}$ is extended linearly to the group ring $\mathbb{Z}[G]$. The following formula is a consequence of the orthogonality properties of characters.

Proposition 2.1 (Fourier inversion formula). Let $G$ be an abelian group and let $A=$ $\sum_{g \in G} c_{g} g \in \mathbb{Z}[G]$. Then

$$
c_{g}=\frac{1}{|G|} \sum_{\chi \in \widehat{G}} \chi(A) \overline{\chi(g)} \text { for each } g \in G \text {. }
$$

Suppose $\left\{D_{1}, D_{2}, \ldots, D_{m}\right\}$ is a nontrivial $(v, m, k, \lambda)$-SEDF in a group $G$, and write $D=\bigcup_{i=1}^{m} D_{i}$. Then (1.2) is equivalent to

$$
D_{j}\left(D^{(-1)}-D_{j}^{(-1)}\right)=\lambda(G-1) \text { in } \mathbb{Z}[G] \text { for each } j \text { satisfying } 1 \leq j \leq m
$$

Apply a nonprincipal character $\chi \in \widehat{G}$ to obtain

$$
\chi\left(D_{j}\right)\left(\overline{\chi(D)-\chi\left(D_{j}\right)}\right)=-\lambda \text { for all nonprincipal } \chi \in \widehat{G} \text { and for each } j .
$$

Some basic restrictions were derived from (2.1) in [21, Lemma 3.1]. We now extend that analysis.

It follows from (2.1) that for each $j$ satisfying $1 \leq j \leq m$,

$$
\left|\chi\left(D_{j}\right)\right|^{2}=\lambda \text { if and only if } \chi(D)=0 .
$$

Define

$$
\begin{aligned}
\widehat{G}^{0} & =\{\text { nonprincipal } \chi \in \widehat{G} \mid \chi(D)=0\}, \\
\widehat{G}^{N} & =\{\text { nonprincipal } \chi \in \widehat{G} \mid \chi(D) \neq 0\},
\end{aligned}
$$

so that $\widehat{G}$ may be partitioned (with respect to $D$ ) as the disjoint union $\left\{\chi_{0}\right\} \cup \widehat{G}^{0} \cup \widehat{G}^{N}$. We now show that the set $\widehat{G}^{N}$ is nonempty.

Lemma 2.2 ([21, Lemma 3.1 (d)]) Suppose $\left\{D_{1}, D_{2}, \ldots, D_{m}\right\}$ is a nontrivial $(v, m, k, \lambda)-S E D F$ in a group $G$, and let $D=\bigcup_{i=1}^{m} D_{i}$. Then $\left|\widehat{G}^{N}\right|>0$. 
Proof Suppose, for a contradiction, that $\chi(D)=0$ for each nonprincipal $\chi \in \widehat{G}$. Write $D=\sum_{g \in G} c_{g} g$ in $\mathbb{Z}[G]$ and use Proposition 2.1 to show that for each $g \in G$ we have

$$
c_{g}=\frac{1}{v} \chi_{0}(D) \overline{\chi_{0}(g)}=\frac{k m}{v} .
$$

By Lemma 1.5 we have $v>k m$, giving the contradiction $0<c_{g}<1$.

For each $\chi \in \widehat{G}^{N}$, set $\alpha_{j, \chi}$ to be the real number $\frac{\left|\chi\left(D_{j}\right)\right|^{2}}{\left|\chi\left(D_{j}\right)\right|^{2}-\lambda}$. Then conjugate (2.1), multiply both sides by $\chi\left(D_{j}\right)$, and rearrange to give

$$
\chi\left(D_{j}\right)=\alpha_{j, \chi} \chi(D) \text { for } \chi \in \widehat{G}^{N} .
$$

Substitute for $\chi\left(D_{j}\right)$ from (2.4) into (2.1) to obtain a quadratic equation in $\alpha_{j, \chi}$ :

$$
\alpha_{j, \chi}^{2}-\alpha_{j, \chi}-\frac{\lambda}{|\chi(D)|^{2}}=0 \text { for } \chi \in \widehat{G}^{N} .
$$

The solutions of this equation are

$$
\alpha_{\chi}^{+}=\frac{1}{2}\left(1+\sqrt{1+\frac{4 \lambda}{|\chi(D)|^{2}}}\right), \quad \alpha_{\chi}^{-}=\frac{1}{2}\left(1-\sqrt{1+\frac{4 \lambda}{|\chi(D)|^{2}}}\right) \text { for } \chi \in \widehat{G}^{N} .
$$

For each $\chi \in \widehat{G}^{N}$, let $\ell_{\chi}^{+}$and $\ell_{\chi}^{-}$be the number of times $\alpha_{j, \chi}$ takes the value $\alpha_{\chi}^{+}$and $\alpha_{\chi}^{-}$, respectively, as $j$ ranges over $1 \leq j \leq m$. Using $\chi(D)=\sum_{j=1}^{m} \chi\left(D_{j}\right)$, we find from (2.4) that

$$
\ell_{\chi}^{+} \alpha_{\chi}^{+}+\ell_{\chi}^{-} \alpha_{\chi}^{-}=1
$$

Combine with the counting condition $\ell_{\chi}^{+}+\ell_{\chi}^{-}=m$ to determine $\ell_{\chi}^{+}$and $\ell_{\chi}^{-}$as

$$
\ell_{\chi}^{+}=\frac{m}{2}-\frac{m-2}{2 \sqrt{1+\frac{4 \lambda}{|\chi(D)|^{2}}}}, \quad \ell_{\chi}^{-}=\frac{m}{2}+\frac{m-2}{2 \sqrt{1+\frac{4 \lambda}{|\chi(D)|^{2}}}} \quad \text { for } \chi \in \widehat{G}^{N} .
$$

In particular, $\ell_{\chi}^{+} \geq \frac{m}{2}-\frac{m-2}{2}=1$ and $\ell_{\chi}^{-} \geq 1$, so the values $\alpha_{\chi}^{+}$and $\alpha_{\chi}^{-}$both occur as $j$ ranges over $\{1,2, \ldots, m\}$. Therefore from (2.4) we have

$$
\left\{\chi\left(D_{j}\right) \mid 1 \leq j \leq m\right\}=\left\{\alpha_{\chi}^{+} \chi(D), \alpha_{\chi}^{-} \chi(D)\right\} \text { for } \chi \in \widehat{G}^{N} .
$$

The expressions (2.7) illustrate a fundamental difference between the cases $m=2$ and $m>2$. When $m=2$, these expressions reduce to $\ell_{\chi}^{+}=\ell_{\chi}^{-}=1$. But when $m>2$, we require $\sqrt{1+\frac{4 \lambda}{|\chi(D)|^{2}}} \in \mathbb{Q}$ for each $\chi \in \widehat{G}^{N}$ in order for $\ell_{\chi}^{+}$and $\ell_{\chi}^{-}$to be integers. We shall see in Sect. 5 that this yields strong restrictions on the character values of $\chi(D)$ and $\chi\left(D_{j}\right)$ for SEDFs when $m>2$, which do not apply when $m=2$.

We conclude this section with a result required in Sect. 3. 
Lemma 2.3 Suppose $\left\{D_{1}, D_{2}, \ldots, D_{m}\right\}$ is a nontrivial $(v, m, k, \lambda)-S E D F$ in a group $G$, where $1 \notin \bigcup_{i=1}^{m} D_{i}$. Then, for each $j$, neither $D_{j} \cup\{1\}$ nor $G \backslash D_{j}$ is a subgroup of $G$.

Proof Suppose, for a contradiction, that $D_{j} \cup\{1\}$ is a subgroup of $G$. Since $m \geq 2$, there exists a nonprincipal character $\chi$ of $G$ which is principal on $D_{j} \cup\{1\}$. Then (2.1) gives $k(\overline{\chi(D)}-k)=-\lambda$, so that $\chi(D)=k-\frac{\lambda}{k}$ is a rational number. Since $\chi(D)$ is also an algebraic integer, $\lambda / k$ is an integer and therefore $\lambda \geq k$. This contradicts Proposition 1.4 (6).

Suppose, for a contradiction, that $G \backslash D_{j}$ is a subgroup of $G$. Then $(v-k) \mid v$, and since $k>1$ we have $k \geq \frac{v}{2}$. But $v>k m$ and $m \geq 2$ gives the contradiction $k<\frac{v}{2}$.

\section{Near-complete SEDFs}

Let $D$ be a $k$-subset of a group $G$ of order $v$, where $1 \notin D$. The subset $D$ is a $(v, k, \lambda, \mu)$ partial difference set (PDS) in $G$ if

$$
D D^{(-1)}=(k-\mu) \cdot 1+\lambda D+\mu(G-D) \text { in } \mathbb{Z}[G] .
$$

(A slightly different definition, which we will not require, applies when $1 \in D$.) The PDS $D$ is regular if $D=D^{(-1)}$, and is trivial if either $D \cup\{1\}$ or $G \backslash D$ is a subgroup of $G$. In this section we prove the following result, which characterizes the parameters of a nontrivial near-complete $(v, m, k, \lambda)$-SEDF and provides the first known example of a nontrivial $(v, m, k, \lambda)$-SEDF having $m>2$.

Theorem 3.1 Let $D_{1}, D_{2}, \ldots, D_{m}$ partition the nonidentity elements of an abelian group $G$ of order $v=k m+1$ into $m$ subsets each of size $k>1$. Then $\left\{D_{1}, D_{2}, \ldots, D_{m}\right\}$ is a nontrivial near-complete $(v, m, k, \lambda)-S E D F$ in $G$ if and only if either

(1) $(v, m, k, \lambda)=\left(v, 2, \frac{v-1}{2}, \frac{v-1}{4}\right)$ and $v \equiv 1(\bmod 4)$ and $D_{1}$ is a nontrivial regular $\left(v, \frac{v-1}{2}, \frac{v-5}{4}, \frac{v-1}{4}\right)-P D S$ in $G$, or

(2) $(v, m, k, \lambda)=(243,11,22,20)$ and each $D_{j}$ is a nontrivial regular $(243,22,1$, 2)-PDS in $G$ for $1 \leq j \leq 11$.

Furthermore, a $(243,11,22,20)$-SEDF exists in $\mathbb{Z}_{3}^{5}$.

The restriction of Theorem 3.1 to the case $m=2$ is due to Huczynska and Paterson [15, Theorem 4.6], and also to Ding [12, Proposition 2.1] from the viewpoint of difference families. One direction of the case $m=2$, namely the construction of an SEDF from a PDS, was also proved in [11, Section 3]. Necessary and sufficient conditions for the existence of a PDS with the parameters specified in (1) and (2) of Theorem 3.1 are not known. However, sufficient conditions for the existence of a PDS with the parameters specified in (1) of Theorem 3.1 (known as a Paley-type PDS) are known to include: $G$ is elementary abelian and $v$ is a prime power congruent to 1 modulo 4 [24]; $G=\mathbb{Z}_{p^{r}}^{2}$ for an odd prime $p$ [17]; and $G=\mathbb{Z}_{3}^{2} \times \mathbb{Z}_{p}^{4 r}$ for an odd prime $p$ [26]. Necessary conditions for the existence of a PDS in an abelian group $G$ with the parameters specified in (2) of Theorem 3.1 are that $G=\mathbb{Z}_{3}^{5}, \mathbb{Z}_{3}^{3} \times \mathbb{Z}_{9}$, or $\mathbb{Z}_{3} \times \mathbb{Z}_{9}^{2}$ [20, Theorem 6.9]; existence is known for $G=\mathbb{Z}_{3}^{5}$ [4], [6, Section 10]. 
In order to establish Theorem 3.1, we make the following connection between a nontrivial near-complete SEDF and a collection of nontrivial regular PDSs.

Lemma 3.2 Let $D_{1}, D_{2}, \ldots, D_{m}$ partition the nonidentity elements of an abelian group $G$ of order $v=k m+1$ into $m$ subsets each of size $k>1$. Then $\left\{D_{1}, D_{2}, \ldots, D_{m}\right\}$ is a nontrivial near-complete $(v, m, k, \lambda)-S E D F$ in $G$ if and only if each $D_{j}$ is a nontrivial regular $(v, k, k-\lambda-1, k-\lambda)-P D S$ in $G$ for $1 \leq j \leq m$.

Proof Since $D_{1}, D_{2}, \ldots, D_{m}$ is a partition of the nonidentity elements of $G$, for each $j$ satisfying $1 \leq j \leq m$ we have $1 \notin D_{j}$ and

$$
\sum_{\substack{1 \leq i \leq m \\ i \neq j}} D_{i}=G-D_{j}-1
$$

and therefore

$$
D_{j} \sum_{\substack{1 \leq i \leq m \\ i \neq j}} D_{i}^{(-1)}=D_{j}\left(G-D_{j}^{(-1)}-1\right)=k G-D_{j} D_{j}^{(-1)}-D_{j} .
$$

It follows that $\left\{D_{1}, D_{2}, \ldots, D_{m}\right\}$ is a nontrivial near-complete $(v, m, k, \lambda)$-SEDF in $G$ if and only if, for each $j$,

$$
\lambda(G-1)=k G-D_{j} D_{j}^{(-1)}-D_{j}
$$

which rearranges to

$$
D_{j} D_{j}^{(-1)}=\lambda \cdot 1+(k-\lambda-1) D_{j}+(k-\lambda)\left(G-D_{j}\right)
$$

Equivalently, each $D_{j}$ is a $(v, k, k-\lambda-1, k-\lambda)$-PDS in $G$.

To complete the proof, we require that if $\left\{D_{1}, D_{2}, \ldots, D_{m}\right\}$ is a nontrivial nearcomplete $(v, m, k, \lambda)$-SEDF in $G$, then each $\operatorname{PDS} D_{j}$ is nontrivial and regular. Nontriviality of each $D_{j}$ is given by Lemma 2.3, and regularity by [20, Proposition 1.2].

The parameters of the nontrivial regular PDSs specified in Lemma 3.2 take the form $(v, k, \mu-1, \mu)$. The following result characterizes all such parameters when the group is abelian.

Theorem 3.3 [2]; see also [20, Theorem 13.1]) Suppose there exists a nontrivial regular $(v, k, \mu-1, \mu)-P D S$ in an abelian group. Then either

(1) $(v, k, \mu-1, \mu)=\left(v, \frac{v-1}{2}, \frac{v-5}{4}, \frac{v-1}{4}\right)$ and $v \equiv 1(\bmod 4)$, or

(2) $(v, k, \mu-1, \mu)=(243,22,1,2)$ or $(243,220,199,220)$.

We can now give the structure of the proof of Theorem 3.1. 
Proof of Theorem 3.1 By Lemma 3.2, $\left\{D_{1}, D_{2}, \ldots, D_{m}\right\}$ is a nontrivial near-complete $(v, m, k, \lambda)$-SEDF in $G$ if and only if each $D_{j}$ is a nontrivial regular $(v, k, k-\lambda-$ $1, k-\lambda)$-PDS in $G$ for $1 \leq j \leq m$. Since $m=(v-1) / k$, by Theorem 3.3 this holds if and only if either

(1) $(v, m, k, \lambda)=\left(v, 2, \frac{v-1}{2}, \frac{v-1}{4}\right)$ and $v \equiv 1(\bmod 4)$ and each of $D_{1}, D_{2}$ is a nontrivial regular $\left(v, \frac{v-1}{2}, \frac{v-5}{4}, \frac{v-1}{4}\right)$-PDS in $G$, or

(2) $(v, m, k, \lambda)=(243,11,22,20)$ and each $D_{j}$ is a nontrivial regular $(243,22,1,2)$ PDS in $G$ for $1 \leq j \leq 11$.

For case (1), the desired result follows from the observation that if $D_{1}$ is a regular $\left(v, \frac{v-1}{2}, \frac{v-5}{4}, \frac{v-1}{4}\right)$-PDS in $G$ that does not contain the identity, then so is $D_{2}=$ $G \backslash\left(D_{1} \cup\{1\}\right)$ [15, Lemma 4.3].

It remains to construct a $(243,11,22,20)-S E D F$ in $\mathbb{Z}_{3}^{5}$, which is carried out below.

In the rest of this section we shall construct a $(243,11,22,20)$-SEDF in $\mathbb{Z}_{3}^{5}$, regarded as the additive group of $\mathbb{F}_{3}^{5}$. By Lemma 3.2, this is equivalent to partitioning the nonzero elements of $\mathbb{F}_{3}^{5}$ into 11 subsets, each of which is a nontrivial regular $(243,22,1,2)$-PDS in the additive group of $\mathbb{F}_{3}^{5}$.

We firstly review the construction of a single nontrivial regular $(243,22,1,2)$-PDS in the additive group of $\mathbb{F}_{3}^{5}$. This PDS was originally constructed from the perfect ternary Golay code [4]; we shall use the following alternative description involving a group of collineations of projective space having exactly two point-orbits [6, Section 10]. The $\frac{3^{5}-1}{3-1}=121$ points of the projective space $\operatorname{PG}(4,3)$ are the 1-dimensional subspaces of the vector space $\mathbb{F}_{3}^{5}$ over $\mathbb{F}_{3}$. Each such point has the form $\langle x\rangle$ for some nonzero $x \in \mathbb{F}_{3}^{5}$, and corresponds to the vectors $x$ and $2 x$ of $\mathbb{F}_{3}^{5}$. The general linear group $\operatorname{GL}(5,3)$ is the group of $5 \times 5$ invertible matrices over $\mathbb{F}_{3}$, and its center is $Z=\{I, 2 I\}$ where $I$ is the $5 \times 5$ identity matrix. The projective linear group $\operatorname{PGL}(5,3)$ is the quotient group $\operatorname{GL}(5,3) / Z$. The action of an element $A \in \operatorname{PGL}(5,3)$ on a point $\langle x\rangle \in \mathrm{PG}(4,3)$ is given by

$$
A:\langle x\rangle \mapsto\langle x A\rangle,
$$

where $x A$ is the usual vector-matrix product, and this action is transitive on the points of PG(4, 3) [13, p. 57]. Now PGL(5, 3) contains a subgroup of order 7920 which is a representation of the Mathieu group $M_{11}$. The group $M_{11}$ has exactly two pointorbits on PG(4,3): one of size 11 and the other of size 110 [6, Example RT6]. The 22 vectors of $\mathbb{F}_{3}^{5}$ corresponding to the point-orbit of size 11 form a nontrivial regular $(243,22,1,2)-P D S$ in the additive group of $\mathbb{F}_{3}^{5}[6$, Theorem 3.2 and Figure 2b].

Define the elements of $\operatorname{PGL}(5,3)$ :

$$
X=\left[\begin{array}{lllll}
0 & 2 & 1 & 0 & 0 \\
2 & 1 & 1 & 2 & 2 \\
0 & 1 & 1 & 2 & 2 \\
1 & 0 & 2 & 2 & 1 \\
1 & 2 & 2 & 2 & 0
\end{array}\right] \text { and } Y=\left[\begin{array}{lllll}
0 & 0 & 2 & 0 & 2 \\
1 & 1 & 2 & 2 & 0 \\
2 & 2 & 2 & 2 & 2 \\
1 & 2 & 1 & 1 & 0 \\
2 & 2 & 0 & 2 & 1
\end{array}\right]
$$


which satisfy $X^{2}=Y^{4}=(X Y)^{11}=I$. The group $M_{11}$ may be represented explicitly [1] as

$$
M_{11}=\langle X, Y\rangle \text {. }
$$

The software package Magma gives the point-orbit of size 11 under the action of $M_{11}$ on $\mathrm{PG}(4,3)$ as

$$
\begin{aligned}
O_{1}=\{ & \langle(1,0,0,0,0)\rangle,\langle(1,1,0,0,2)\rangle,\langle(2,2,1,0,1)\rangle, \\
& \langle(1,0,2,1,0)\rangle,\langle(0,0,2,1,2)\rangle,\langle(0,1,2,0,0)\rangle, \\
& \langle(0,0,1,0,1)\rangle,\langle(2,0,0,2,1)\rangle,\langle(2,2,1,2,0)\rangle, \\
& \langle(0,1,0,1,2)\rangle,\langle(0,2,0,2,0)\rangle\},
\end{aligned}
$$

and the corresponding nontrivial regular $(243,22,1,2)-P D S$ is

$$
\begin{aligned}
B_{1}=\{ & \left.x \mid\langle x\rangle \in O_{1}\right\} \cup\left\{2 x \mid\langle x\rangle \in O_{1}\right\} \\
= & (1,0,0,0,0),(1,1,0,0,2),(2,2,1,0,1), \\
& (1,0,2,1,0),(0,0,2,1,2),(0,1,2,0,0), \\
& (0,0,1,0,1),(2,0,0,2,1),(2,2,1,2,0), \\
& (0,1,0,1,2),(0,2,0,2,0),(2,0,0,0,0), \\
& (2,2,0,0,1),(1,1,2,0,2),(2,0,1,2,0), \\
& (0,0,1,2,1),(0,2,1,0,0),(0,0,2,0,2), \\
& (1,0,0,1,2),(1,1,2,1,0),(0,2,0,2,1),(0,1,0,1,0)\}
\end{aligned}
$$

in the additive group of $\mathbb{F}_{3}^{5}$.

It is convenient to write

$$
W=X Y=\left[\begin{array}{lllll}
1 & 1 & 0 & 0 & 2 \\
0 & 2 & 1 & 1 & 2 \\
0 & 2 & 0 & 1 & 1 \\
2 & 1 & 2 & 2 & 1 \\
2 & 1 & 0 & 1 & 0
\end{array}\right]
$$

giving the alternative representation

$$
M_{11}=\langle W, Y\rangle
$$

Now the cyclic group $\langle W\rangle$ is an order 11 subgroup of $M_{11}$. The orbit of $\langle(1,0,0,0,0)\rangle$ under the action of $\langle W\rangle$ has size 1 or 11 ; since $\langle W\rangle$ does not fix the point $\langle(1,0,0,0,0)\rangle$, this orbit is the whole of $O_{1}$ :

$$
O_{1}=\{\langle(1,0,0,0,0) A\rangle \mid A \in\langle W\rangle\} .
$$


Recall that the group $M_{11}$ has exactly two point-orbits on $\mathrm{PG}(4,3)$ : one of size 11 (the set $\left.O_{1}\right)$, and the other of size 110 . We will show that the action of the cyclic subgroup $\langle W\rangle$ of $M_{11}$ on the points of $\mathrm{PG}(4,3)$ breaks the point-orbit of size 110 (under the action of $M_{11}$ ) into 10 point-orbits of size 11 , each of which also corresponds to a nontrivial regular $(243,22,1,2)$-PDS in the additive group of $\mathbb{F}_{3}^{5}$. This will give the partition of the nonzero elements of $\mathbb{F}_{3}^{5}$ into 11 subsets required under Lemma 3.2.

The centralizer of $W$ in $\operatorname{PGL}(5,3)$ is the group $C(W)=\{B \in \operatorname{PGL}(5,3): B W=$ $W B\}$. Magma gives $C(W)$ to be a cyclic group of order 121 , one of whose generators is

$$
S=\left[\begin{array}{lllll}
1 & 2 & 2 & 1 & 2 \\
1 & 2 & 0 & 1 & 2 \\
0 & 2 & 2 & 1 & 2 \\
0 & 0 & 0 & 0 & 2 \\
1 & 1 & 1 & 0 & 1
\end{array}\right]
$$

which satisfies $W=S^{11}$. Define subsets $O_{2}, O_{3}, \ldots, O_{11}$ of $\operatorname{PG}(4,3)$ by

$$
O_{j}=\left\{\left\langle x S^{j-1}\right\rangle \mid\langle x\rangle \in O_{1}\right\} \text { for } 2 \leq j \leq 11
$$

Then for $1 \leq j \leq 11$ we find from (3.5) that

$$
\begin{aligned}
O_{j} & =\left\{\left\langle(1,0,0,0,0) A S^{j-1}\right\rangle \mid A \in\langle W\rangle\right\} \\
& =\left\{\left\langle(1,0,0,0,0) S^{j-1} A\right\rangle \mid A \in\langle W\rangle\right\}
\end{aligned}
$$

because $S \in C(W)$, and therefore the subset $O_{j}$ is the size 11 orbit of the point $\left\langle(1,0,0,0,0) S^{j-1}\right\rangle$ under the action of $\langle W\rangle$. Furthermore, using $W=S^{11}$ we may write

$$
O_{j}=\left\{\left\langle(1,0,0,0,0) S^{11 i+j-1}\right\rangle \mid 0 \leq i \leq 10\right\} \text { for } 1 \leq j \leq 11,
$$

so that

$$
\bigcup_{j=1}^{11} O_{j}=\left\{\left\langle(1,0,0,0,0) S^{\ell}\right\rangle \mid 0 \leq \ell \leq 120\right\} .
$$

We claim that the subsets $O_{1}, O_{2}, \ldots, O_{11}$ form a partition of the 121 points of $\mathrm{PG}(4,3)$. Suppose, for a contradiction, that there is an integer $n$ satisfying $1 \leq n \leq 120$ such that

$$
\left\langle(1,0,0,0,0) S^{n}\right\rangle=\langle(1,0,0,0,0)\rangle .
$$

Since $\langle S\rangle=C(W)$ has order 121 , the matrix $S^{n}$ has order 11 or 121 . But $S^{n}$ cannot have order 121, otherwise $S$ would fix the point $\langle(1,0,0,0,0)\rangle$ and then from (3.8) we would have $\bigcup_{j=1}^{11} O_{j}=\{\langle(1,0,0,0,0)\rangle\}$, contradicting (3.3). Therefore $S^{n}$ has order 11 , so $S^{n}=S^{11 i}$ for some $i$ satisfying $1 \leq i \leq 10$. But from (3.7) the 11 points $\left\{\left\langle(1,0,0,0,0) S^{11 i}\right\rangle \mid 0 \leq i \leq 10\right\}$ comprise the orbit $O_{1}$, and from (3.3) these 11 points are all distinct. This contradicts (3.9) and establishes the claim. 
Finally, define subsets $B_{2}, B_{3}, \ldots, B_{11}$ of the nonzero elements of $\mathbb{F}_{3}^{5}$ by setting

$$
B_{j}=\left\{x \mid\langle x\rangle \in O_{j}\right\} \cup\left\{2 x \mid\langle x\rangle \in O_{j}\right\} \text { for } 2 \leq j \leq 11 .
$$

The subsets $B_{1}, B_{2}, \ldots, B_{11}$ partition the 242 nonzero elements of $\mathbb{F}_{3}^{5}$, and from (3.4) and (3.6) we have

$$
B_{j}=\left\{x S^{j-1} \mid x \in B_{1}\right\} \text { for } 1 \leq j \leq 11 .
$$

Moreover, $B_{1}$ is a nontrivial regular $(243,22,1,2)$-PDS in the additive group of $\mathbb{F}_{3}^{5}$, so from the definition (3.1) the multiset $\left\{x-y \mid x, y \in B_{1}\right\}$ contains the element 0 exactly 22 times, each element of $B_{1}$ exactly once, and each other element of $\mathbb{F}_{3}^{5}$ exactly twice. Since $S$ is invertible, it follows from (3.10) that each $B_{j}$ is also a nontrivial regular $(243,22,1,2)-P D S$ in the additive group of $\mathbb{F}_{3}^{5}$ for $2 \leq j \leq 11$. By Lemma 3.2, $\left\{B_{1}, B_{2}, \ldots, B_{11}\right\}$ is therefore a $(243,11,22,20)$ near-complete SEDF in the additive group of $\mathbb{F}_{3}^{5}$.

Explicitly, we have

$$
\begin{aligned}
& B_{2}=\{(1,2,2,1,2),(1,0,1,2,0),(2,2,1,2,2), \\
& (1,0,0,0,2),(2,0,0,2,2),(1,0,1,0,0) \text {, } \\
& (1,0,0,1,0),(0,2,2,2,0),(1,1,0,2,2) \text {, } \\
& (0,1,2,1,0),(2,1,0,2,2),(2,1,1,2,1) \text {, } \\
& (2,0,2,1,0),(1,1,2,1,1),(2,0,0,0,1) \text {, } \\
& (1,0,0,1,1),(2,0,2,0,0),(2,0,0,2,0) \text {, } \\
& (0,1,1,1,0),(2,2,0,1,1),(0,2,1,2,0),(1,2,0,1,1)\} \text {, } \\
& B_{3}=\{(2,0,2,2,2),(1,1,1,2,2),(0,0,2,2,1), \\
& (0,1,1,1,1),(1,0,0,2,1),(1,1,1,2,1) \text {, } \\
& (1,2,2,1,1),(2,2,1,1,0),(1,0,1,2,1) \text {, } \\
& (1,0,1,0,2),(2,2,0,0,0),(1,0,1,1,1) \text {, } \\
& (2,2,2,1,1),(0,0,1,1,2),(0,2,2,2,2) \text {, } \\
& (2,0,0,1,2),(2,2,2,1,2),(2,1,1,2,2) \text {, } \\
& (1,1,2,2,0),(2,0,2,1,2),(2,0,2,0,1),(1,1,0,0,0)\} \text {, } \\
& B_{4}=\{(1,1,1,1,2),(1,2,0,0,0),(1,2,2,2,0) \text {, } \\
& (2,2,0,2,1),(2,0,0,1,1),(0,1,2,0,2), \\
& (1,2,1,2,1),(1,1,0,2,0),(2,2,2,2,0) \text {, } \\
& (0,0,0,2,0),(1,2,1,1,2),(2,2,2,2,1) \text {, } \\
& (2,1,0,0,0),(2,1,1,1,0),(1,1,0,1,2) \text {, } \\
& (1,0,0,2,2),(0,2,1,0,1),(2,1,2,1,2) \text {, } \\
& (2,2,0,1,0),(1,1,1,1,0),(0,0,0,1,0),(2,1,2,2,1)\}, \\
& B_{5}=\{(1,2,0,0,1),(0,0,2,0,0),(0,1,0,2,2), \\
& (2,0,2,1,1),(0,2,2,2,1),(0,2,0,0,2),
\end{aligned}
$$




$$
\begin{aligned}
& (1,0,2,1,1),(2,1,2,2,2),(1,0,2,0,1) \text {, } \\
& (0,0,0,0,1),(2,1,0,1,0),(2,1,0,0,2) \text {, } \\
& (0,0,1,0,0),(0,2,0,1,1),(1,0,1,2,2) \text {, } \\
& (0,1,1,1,2),(0,1,0,0,1),(2,0,1,2,2) \text {, } \\
& (1,2,1,1,1),(2,0,1,0,2),(0,0,0,0,2),(1,2,0,2,0)\} \text {, } \\
& B_{6}=\{(1,1,0,0,1),(0,1,1,2,1),(0,1,2,1,2) \text {, } \\
& (0,0,0,1,2),(0,0,2,1,1),(1,0,2,2,0), \\
& (2,1,1,0,0),(2,0,1,2,1),(2,1,1,0,1) \text {, } \\
& (1,1,1,0,1),(0,0,1,0,2),(2,2,0,0,2) \text {, } \\
& (0,2,2,1,2),(0,2,1,2,1),(0,0,0,2,1) \text {, } \\
& (0,0,1,2,2),(2,0,1,1,0),(1,2,2,0,0) \text {, } \\
& (1,0,2,1,2),(1,2,2,0,2),(2,2,2,0,2),(0,0,2,0,1)\} \text {, } \\
& B_{7}=\{(0,2,0,2,2),(2,2,0,2,0),(0,2,0,0,1), \\
& (2,2,2,0,1),(1,2,2,2,1),(1,0,0,0,1) \text {, } \\
& (0,2,0,1,2),(0,1,1,0,2),(1,0,1,1,0) \text {, } \\
& (0,1,2,0,1),(2,1,1,1,1),(0,1,0,1,1) \text {, } \\
& (1,1,0,1,0),(0,1,0,0,2),(1,1,1,0,2) \text {, } \\
& (2,1,1,1,2),(2,0,0,0,2),(0,1,0,2,1) \text {, } \\
& (0,2,2,0,1),(2,0,2,2,0),(0,2,1,0,2),(1,2,2,2,2)\} \text {, } \\
& B_{8}=\{(1,0,2,2,1),(1,2,1,1,0),(0,2,1,2,2) \text {, } \\
& (2,1,0,0,1),(1,2,1,2,0),(2,0,0,1,0) \text {, } \\
& (1,0,2,2,2),(0,0,1,2,0),(1,1,1,2,0) \text {, } \\
& (2,1,2,0,1),(1,0,1,1,2),(2,0,1,1,2) \text {, } \\
& (2,1,2,2,0),(0,1,2,1,1),(1,2,0,0,2) \text {, } \\
& (2,1,2,1,0),(1,0,0,2,0),(2,0,1,1,1) \text {, } \\
& (0,0,2,1,0),(2,2,2,1,0),(1,2,1,0,2),(2,0,2,2,1)\} \text {, } \\
& B_{9}=\{(2,1,1,0,2),(0,2,1,1,1),(1,2,1,0,0), \\
& (1,1,2,0,1),(0,2,1,1,0),(2,1,1,2,0) \text {, } \\
& (0,2,2,0,0),(0,2,2,1,0),(2,0,1,0,1) \text {, } \\
& (1,2,0,2,2),(0,0,0,2,2),(1,2,2,0,1) \text {, } \\
& (0,1,2,2,2),(2,1,2,0,0),(2,2,1,0,2) \text {, } \\
& (0,1,2,2,0),(1,2,2,1,0),(0,1,1,0,0) \text {, } \\
& (0,1,1,2,0),(1,0,2,0,2),(2,1,0,1,1),(0,0,0,1,1)\} \text {, } \\
& B_{10}=\{(2,1,2,1,1),(0,1,0,0,0),(0,2,1,1,2) \text {, } \\
& (0,0,1,1,0),(2,0,2,0,2),(0,2,0,1,0) \text {, } \\
& (2,2,1,1,2),(2,2,1,1,1),(0,1,1,0,1) \text {, } \\
& (2,2,1,0,0),(2,2,2,0,0),(1,2,1,2,2) \text {, }
\end{aligned}
$$




$$
\begin{aligned}
& (0,2,0,0,0),(0,1,2,2,1),(0,0,2,2,0), \\
& (1,0,1,0,1),(0,1,0,2,0),(1,1,2,2,1), \\
& (1,1,2,2,2),(0,2,2,0,2),(1,1,2,0,0),(1,1,1,0,0)\}, \\
B_{11}=\{( & (1,2,0,2,1),(1,2,0,1,2),(1,2,1,0,1), \\
& (0,2,2,1,1),(1,1,1,1,1),(2,1,0,2,0), \\
& (0,0,2,2,2),(2,2,1,2,1),(2,2,0,2,2), \\
& (1,1,0,2,1),(1,0,2,0,0),(2,1,0,1,2), \\
& (2,1,0,2,1),(2,1,2,0,2),(0,1,1,2,2), \\
& (2,2,2,2,2),(1,2,0,1,0),(0,0,1,1,1), \\
& (1,1,2,1,2),(1,1,0,1,1),(2,2,0,1,2),(2,0,1,0,0)\} .
\end{aligned}
$$

\section{An exponent bound and its application}

In this section, we present an exponent bound on a group $G$ containing a $(v, m, k, \lambda)$ $\mathrm{SEDF}$, and use it to prove nonexistence results for the case $m=2$.

Let $G=H \times L$ be an abelian group. Each element of $G$ can be expressed uniquely as $h \ell$ for $h \in H$ and $\ell \in L$, and the natural projection $\rho$ from $G$ to $H$ is defined by $\rho(h \ell)=h$. Each $\tilde{\chi} \in \widehat{H}$ induces a lifting character $\chi \in \widehat{G}$ satisfying $\chi(g)=\tilde{\chi}(\rho(g))$ for every $g \in G$. From now on, we shall use $G_{p}$ to denote the Sylow $p$-subgroup of the group $G$, where $p$ is a prime. For a positive integer $n$, we use $\zeta_{n}$ to denote the primitive $n$-th complex root of unity $e^{2 \pi i / n}$.

A prime $p$ is a primitive root modulo $n$ if $p$ is a generator of the multiplicative group of integers modulo $n$. A prime $p$ is self-conjugate modulo $n$ if there is an integer $j$ for which $p^{j} \equiv-1\left(\bmod n_{p}\right)$, where $n_{p}$ is the largest divisor of $n$ that is not divisible by $p$. If a prime $p$ is a primitive root modulo $n$, then $p$ is self-conjugate modulo $n$. For $X \in \mathbb{Z}\left[\zeta_{n}\right]$, we use $(X)$ to denote the principal idea generated by $X$ in $\mathbb{Z}\left[\zeta_{n}\right]$. We begin with a preparatory lemma.

Lemma 4.1 Let $p$ and $q$ be primes, let $q$ be a primitive root modulo $p^{e}$, and let $q^{f} \| u$ for some positive integer $f$. Suppose that $X, X^{\prime} \in \mathbb{Z}\left[\zeta_{p^{e}}\right]$ satisfy $X \overline{X^{\prime}}=u$. Then either $X \equiv 0\left(\bmod q^{\lceil f / 2\rceil}\right)$ or $X^{\prime} \equiv 0\left(\bmod q^{\lceil f / 2\rceil}\right)$. Furthermore, if $X=X^{\prime}$, then $f$ is even.

Proof Since $X \overline{X^{\prime}}=u$ and $q^{f} \| u$, we have $X \overline{X^{\prime}} \equiv 0\left(\bmod q^{f}\right)$. Now $q$ is a primitive root modulo $p^{e}$, so $(q)$ is a prime ideal in $\mathbb{Z}\left[\zeta_{p^{e}}\right]$ [16, Chapter 13, Theorem 2], which we denote by $\mathcal{Q}$. Hence

$$
X \overline{X^{\prime}} \equiv 0 \quad\left(\bmod \mathcal{Q}^{f}\right)
$$

and so

$$
\mathcal{Q}^{f} \mid(X)\left(\overline{X^{\prime}}\right) .
$$


Therefore either $\mathcal{Q}^{\lceil f / 2\rceil} \mid(X)$ or $\mathcal{Q}^{\lceil f / 2\rceil} \mid\left(X^{\prime}\right)$, and so either $X \equiv 0\left(\bmod q^{\lceil f / 2\rceil}\right)$ or $X^{\prime} \equiv 0\left(\bmod q^{\lceil f / 2\rceil}\right)$.

Now suppose $X=X^{\prime}$, so that $\mathcal{Q}^{\lceil f / 2\rceil} \mid(X)$. Since $q$ is a primitive root modulo $p^{e}$, we have that $q$ is self-conjugate modulo $p^{e}$. This implies $\mathcal{Q}$ is invariant under complex conjugation [5, Chapter VI, Corollary 15.5], so that $\mathcal{Q}^{\lceil f / 2\rceil} \mid(\bar{X})$ and therefore $\mathcal{Q}^{2\lceil f / 2\rceil} \mid(X)(\bar{X})$. But $q^{f} \| u$, so $\mathcal{Q}^{f} \|(X)(\bar{X})$. Therefore $f$ is even.

We now prove the following exponent bound.

Theorem 4.2 Suppose there exists a $(v, m, k, \lambda)$-SEDF in a group $G$. Let $p$ and $q$ be primes such that $p^{d} \| v$ and $q^{f} \| \lambda$ for some positive integers $d$ and $f$, and suppose that $q$ is a primitive root modulo $p^{d}$. Let $G_{p}$ be the Sylow $p$-subgroup of $G$. Then

$$
\exp \left(G_{p}\right) \leq v / q^{\lceil f / 2\rceil}
$$

Proof Let $\left\{D_{1}, D_{2}, \ldots, D_{m}\right\}$ be the SEDF, and let $D=\bigcup_{i=1}^{m} D_{i}$. Let $\exp \left(G_{p}\right)=p^{e}$, let $H$ be a cyclic $p$-subgroup of $G$ of order $p^{e}$ occurring as a direct factor of $G$, and let $\rho$ be the natural projection from $G$ to $H$. Let $\tilde{\chi}$ be a generator of $\widehat{H}$, and let $\chi$ be the associated lifting character on $G$. Then

$$
\tilde{\chi}\left(\rho\left(D_{1}\right)\right) \overline{\widetilde{\chi}\left(\rho\left(D-D_{1}\right)\right)}=\chi\left(D_{1}\right) \overline{\chi\left(D-D_{1}\right)}=-\lambda
$$

by (2.1). Now $q$ is a primitive root modulo $p^{d}$, so $q$ is also a primitive root modulo $p^{e}\left[16\right.$, Chapter 4, Lemma 3]. Apply Lemma 4.1 with $X=\tilde{\chi}\left(\rho\left(D_{1}\right)\right)$ and $X^{\prime}=$ $\tilde{\chi}\left(\rho\left(D-D_{1}\right)\right)$ and $u=-\lambda$ to show that there is a subset $D^{\prime}$ of $G$ (either $D_{1}$ or $\left.D \backslash D_{1}\right)$ for which $\tilde{\chi}\left(\rho\left(D^{\prime}\right)\right) \equiv 0\left(\bmod q^{\lceil f / 2\rceil}\right)$. Write

$$
\tilde{\chi}\left(\rho\left(D^{\prime}\right)\right)=\sum_{i=0}^{p^{e-1}-1} d_{i} \zeta_{p^{e}}^{i}
$$

where $d_{i}=\sum_{j=0}^{p-1} c_{i, j} \zeta_{p}^{j}$ and each $c_{i, j} \in \mathbb{Z}$. We have shown that $q^{\lceil f / 2\rceil}$ | $\sum_{i=0}^{p^{e-1}-1} d_{i} \zeta_{p^{e}}^{i}$. Since $\left\{1, \zeta_{p^{e}}, \zeta_{p^{e}}^{2}, \ldots, \zeta_{p^{e}}^{p^{e-1}-1}\right\}$ is a linearly independent set over $\mathbb{Q}\left[\zeta_{p}\right]$, this implies that

$$
q^{\lceil f / 2\rceil} \mid d_{i} \text { for each } i \text {. }
$$

Note that $d_{i}=\sum_{j=0}^{p-1} c_{i, j} \zeta_{p}^{j}=\sum_{j=0}^{p-2}\left(c_{i, j}-c_{i, p-1}\right) \zeta_{p}^{j}$. Because $\left\{1, \zeta_{p}, \zeta_{p}^{2}, \ldots, \zeta_{p}^{p-2}\right\}$ is an integral basis of $\mathbb{Z}\left[\zeta_{p}\right]$, we then obtain

$$
q^{\lceil f / 2\rceil} \mid\left(c_{i, j}-c_{i, p-1}\right) \text { for each } i \text { and } j .
$$

Since $\rho$ is the natural projection from $G$ to $H$, and $|H|=p^{e}$, we have $0 \leq c_{i, j} \leq \frac{v}{p^{e}}$ for each $i$ and $j$, and therefore $-\frac{v}{p^{e}} \leq c_{i, j}-c_{i, p-1} \leq \frac{v}{p^{e}}$ for each $i$ and $j$. Furthermore, from (4.1) and (4.2), $d_{i}^{\prime} \neq 0$ for some $i^{\prime}$ and therefore $c_{i^{\prime}, j^{\prime}}-c_{i^{\prime}, p-1} \neq 0$ for some $j^{\prime}$. It follows from (4.3) that $q^{\lceil f / 2\rceil} \leq v / p^{e}$, or equivalently $p^{e} \leq v / q^{\lceil f / 2\rceil}$. 
Very few nonexistence results for a nontrivial $(v, m, k, \lambda)$-SEDF with $m=2$ are known. We now illustrate the use of Theorem 4.2 by ruling out several families of such parameter sets. When $m=2$ and $k$ is prime, the existence question is already answered: we must have $\lambda=1$ [15, Lemma 3.4], and then by Proposition 1.3 the parameters have the form $\left(k^{2}+1,2, k, 1\right)$. We therefore consider $m=2$ and $k=p_{1} p_{2}$ in Theorem 4.3, where $p_{1}, p_{2}$ are distinct primes and $p_{1}<p_{2}$. The case $\lambda=1$ is dealt with in Proposition 1.3, and the cases $\lambda \geq p_{1} p_{2}$ are ruled out by Proposition 1.4 (6). In view of the counting relation $p_{1}^{2} p_{2}^{2}=\bar{\lambda}(v-1)$ given by (1.3), the remaining cases are $\lambda \in\left\{p_{1}, p_{2}, p_{1}^{2}\right\}$.

Theorem 4.3 Let $p_{1}$ and $p_{2}$ be distinct primes with $p_{1}<p_{2}$.

(1) Let $p$ be a prime such that $p^{d} \| p_{1} p_{2}^{2}+1$ for some positive integer $d$. If $p_{1}$ is a primitive root modulo $p^{d}$ and $p_{2}^{2}+1 \leq p$, then $a\left(p_{1} p_{2}^{2}+1,2, p_{1} p_{2}, p_{1}\right)-S E D F$ does not exist.

(2) Let $p$ be a prime such that $p^{d} \| p_{1}^{2} p_{2}+1$ for some positive integer $d$. If $p_{2}$ is a primitive root modulo $p^{d}$ and $p_{1}^{2}+1 \leq p$, then $a\left(p_{1}^{2} p_{2}+1,2, p_{1} p_{2}, p_{2}\right)-S E D F$ does not exist.

(3) Let $p$ be a prime such that $p^{d} \| p_{2}^{2}+1$ for some positive integer $d$. If $p_{1}$ is a primitive root modulo $p^{d}$ and $p_{2}^{2}+1<p_{1} p$, or if $p_{1}$ is a primitive root modulo $p$ and $p_{2}^{2}+1=p_{1} p$, then $a\left(p_{2}^{2}+1,2, p_{1} p_{2}, p_{1}^{2}\right)-S E D F$ does not exist.

Proof Parts (1), (2) and the first part of (3) are each direct applications of Theorem 4.2, whereas the second part of (3) requires additional arguments; we give the proof for both parts of (3). Suppose, for a contradiction, that $\left\{D_{1}, D_{2}\right\}$ is a $\left(p_{2}^{2}+1,2, p_{1} p_{2}, p_{1}^{2}\right)$ SEDF in a group $G$ of order $p_{2}^{2}+1$.

If $p_{1}$ is a primitive root modulo $p^{d}$ and $p_{2}^{2}+1<p_{1} p$, then by Theorem 4.2 we have $\exp \left(G_{p}\right) \leq \frac{p_{2}^{2}+1}{p_{1}}<p$. This contradicts that $p$ is a prime divisor of $|G|=p_{2}^{2}+1$.

If $p_{1}$ is a primitive root modulo $p$ and $p_{2}^{2}+1=p_{1} p$, then $G=\mathbb{Z}_{p_{1}} \times \mathbb{Z}_{p}$. Let $\rho$ be the natural projection from $G$ to $\mathbb{Z}_{p}$. Let $\widetilde{\chi}$ be a generator of $\widehat{\mathbb{Z}_{p}}$. Then

$$
\tilde{\chi}\left(\rho\left(D_{1}\right)\right) \overline{\widetilde{\chi}\left(\rho\left(D_{2}\right)\right)}=-p_{1}^{2}
$$

by (2.1), so by Lemma 4.1 we may choose $D^{\prime}$ to be one of $D_{1}$ and $D_{2}$ so that $\tilde{\chi}\left(\rho\left(D^{\prime}\right)\right) \equiv 0\left(\bmod p_{1}\right)$. Since $\tilde{\chi}$ is a generator of $\widehat{\mathbb{Z}_{p}}$, there is a generator $h$ of $\mathbb{Z}_{p}$ for which $\tilde{\chi}(h)=\zeta_{p}$. Write $\rho\left(D^{\prime}\right)=\sum_{i=0}^{p-1} d_{i} h^{i}$, where $0 \leq d_{i} \leq p_{1}$ for each $i$, and then

$$
\tilde{\chi}\left(\rho\left(D^{\prime}\right)\right)=\sum_{i=0}^{p-1} d_{i} \zeta_{p}^{i}=\sum_{i=0}^{p-2}\left(d_{i}-d_{p-1}\right) \zeta_{p}^{i}
$$

Since $\tilde{\chi}\left(\rho\left(D^{\prime}\right)\right) \equiv 0\left(\bmod p_{1}\right)$, we have $p_{1} \mid d_{i}-d_{p-1}$ for each $i$. Using $0 \leq d_{i} \leq p_{1}$ for each $i$, we distinguish two cases:

Case 1: $d_{i} \in\left\{0, p_{1}\right\}$ for each $i$ satisfying $0 \leq i \leq p-1$. This gives $\rho\left(D^{\prime}\right)=$ $p_{1} \sum_{i \in I} h^{i}$ for some subset $I$ of $\{0,1, \ldots, p-1\}$, which implies that $D^{\prime}$ is a 
union of cosets of $\mathbb{Z}_{p_{1}}$. But then for a character $\chi \in \widehat{G}$ which is nonprincipal on $\mathbb{Z}_{p_{1}}$ we have $\chi\left(D^{\prime}\right)=0$, contradicting (2.1) because $D^{\prime}=D_{1}$ or $D_{2}$.

Case 2: $d_{0}=d_{1}=\cdots=d_{p-1}$. Then $p$ divides $\left|D^{\prime}\right|=p_{1} p_{2}$, so either $p=p_{1}$ or $p=p_{2}$. Both of these contradict the given conditions on $p, p_{1}, p_{2}$.

Remark 4.4 For example, Theorem 4.3 rules out the existence of a $(v, m, k, \lambda)$-SEDF for

$$
\begin{gathered}
(v, m, k, \lambda) \in\{(19,2,6,2),(26,2,10,4),(46,2,15,5), \\
(118,2,39,13),(122,2,22,4), \\
(154,2,51,17),(172,2,57,19)\} .
\end{gathered}
$$

Theorem 4.2 rules out further parameter sets not excluded by Theorem 4.3 (for which $k$ is not the product of two distinct primes), including

$$
\begin{aligned}
(v, m, k, \lambda) \in\{( & 37,2,12,4),(101,2,20,4),(101,2,30,9), \\
& (101,2,40,16),(122,2,44,16), \\
& (127,2,42,14),(129,2,48,18),(163,2,18,2), \\
& (163,2,36,8),(163,2,54,18), \\
& (163,2,72,32),(177,2,44,11),(181,2,60,20), \\
& (197,2,28,4),(197,2,42,9), \\
& (197,2,56,16),(197,2,70,25),(197,2,84,36)\} .
\end{aligned}
$$

All known examples of a nontrivial $(v, 2, k, \lambda)$-SEDF have $v$ a prime, except for those specified in Proposition 1.1 (1) and (2). The only cases for a $(v, 2, k, \lambda)$-SEDF with $v \leq 50$ that remain open are

$(v, m, k, \lambda) \in\{(28,2,9,3),(33,2,8,2),(49,2,12,3),(50,2,14,4),(50,2,21,9)\}$.

The parameter set $(45,2,22,11)$ does not appear on this list, even though the existence of an SEDF with these parameters in $\mathbb{Z}_{3}^{2} \times \mathbb{Z}_{5}$ is not ruled out by Theorems 4.2 and 4.3: by Theorem 3.1, the existence of an SEDF with these parameters would imply the existence of a nontrivial regular $(45,22,10,11)$-PDS, which is excluded by [20, Corollary 6.3].

\section{SEDFs with $m>2$}

Throughout this section, we suppose that $\left\{D_{1}, D_{2}, \ldots, D_{m}\right\}$ is a nontrivial $(v, m, k, \lambda)$ SEDF in a group $G$ with $m>2$, and write $D=\bigcup_{i=1}^{m} D_{i}$. From (2.7), in order for $\ell_{\chi}^{+}$ and $\ell_{\chi}^{-}$to be integers we must have

$$
\begin{aligned}
& \sqrt{1+\frac{4 \lambda}{|\chi(D)|^{2}}}=\frac{b_{\chi}}{a_{\chi}} \text { for each } \chi \in \widehat{G}^{N}, \quad \text { where } a_{\chi}, b_{\chi} \in \mathbb{Z} \\
& \text { and } b_{\chi}>a_{\chi}>0 \text { and } \operatorname{gcd}\left(a_{\chi}, b_{\chi}\right)=1 .
\end{aligned}
$$


Then (2.7) becomes

$$
\ell_{\chi}^{+}=\frac{m}{2}-\frac{a_{\chi}(m-2)}{2 b_{\chi}}, \quad \ell_{\chi}^{-}=\frac{m}{2}+\frac{a_{\chi}(m-2)}{2 b_{\chi}} \quad \text { for each } \chi \in \widehat{G}^{N} .
$$

It is shown in [3, Lemma 3.3] and [21, Lemma 3.5] that $\left(\ell_{\chi}^{+}, \ell_{\chi}^{-}\right) \notin\{(0, m),(1, m-$ $\left.1),\left(\frac{m}{2}, \frac{m}{2}\right)\right\}$ for $m>2$; this is an immediate consequence of (5.2). Rewrite the expressions (2.6) for $\alpha_{\chi}^{+}$and $\alpha_{\chi}^{-}$using (5.1), and then substitute into (2.8) to obtain

$$
\left\{\chi\left(D_{j}\right) \mid 1 \leq j \leq m\right\}=\left\{\frac{a_{\chi}+b_{\chi}}{2 a_{\chi}} \chi(D), \frac{a_{\chi}-b_{\chi}}{2 a_{\chi}} \chi(D)\right\} \text { for each } \chi \in \widehat{G}^{N} \text {. }
$$

Rearrange (5.1) as

$$
|\chi(D)|^{2}=\frac{4 a_{\chi}^{2} \lambda}{b_{\chi}^{2}-a_{\chi}^{2}} \quad \text { for each } \chi \in \widehat{G}^{N}
$$

and then combine with (5.3) to give

$$
\begin{aligned}
& \left\{\left(|\chi(D)|^{2},\left|\chi\left(D_{j}\right)\right|^{2}\right) \mid 1 \leq j \leq m\right\} \\
& \quad=\left\{\left(\frac{4 a_{\chi}^{2} \lambda}{b_{\chi}^{2}-a_{\chi}^{2}}, \frac{\left(b_{\chi}+a_{\chi}\right) \lambda}{b_{\chi}-a_{\chi}}\right), \quad\left(\frac{4 a_{\chi}^{2} \lambda}{b_{\chi}^{2}-a_{\chi}^{2}}, \frac{\left(b_{\chi}-a_{\chi}\right) \lambda}{b_{\chi}+a_{\chi}}\right)\right\} \text { for each } \chi \in \widehat{G}^{N} .
\end{aligned}
$$

We now derive some divisibility conditions on the values of $a_{\chi}$ and $b_{\chi}$, which restrict the possible values of $|\chi(D)|^{2}$ and $\left|\chi\left(D_{j}\right)\right|^{2}$ via (5.4).

Lemma 5.1 Let $a_{\chi}, b_{\chi}$ be defined as in (5.1) (with reference to the set $D=\bigcup_{i=1}^{m} D_{i}$ associated with a nontrivial $(v, m, k, \lambda)-\operatorname{SEDF}\left\{D_{1}, D_{2}, \ldots, D_{m}\right\}$ in a group $G$ with $m>2$ ). Then

(1) $2 b_{\chi} \mid b_{\chi} m-a_{\chi}(m-2)$, and $b_{\chi} \mid m-2$

(2) $\left(b_{\chi}-a_{\chi}\right) \mid\left(b_{\chi}+a_{\chi}\right) \lambda$, and $\left(b_{\chi}+a_{\chi}\right) \mid\left(b_{\chi}-a_{\chi}\right) \lambda$

(3) $\left(b_{\chi}^{2}-a_{\chi}^{2}\right) \mid 4 \lambda$, and if $b_{\chi}+a_{\chi}$ is odd then $\left(b_{\chi}^{2}-a_{\chi}^{2}\right) \mid \lambda$.

Proof (1) Since $\ell_{\chi}^{+}$is an integer, by (5.2) we have $2 b_{\chi} \mid b_{\chi} m-a_{\chi}(m-2)$. Therefore $b_{\chi} \mid a_{\chi}(m-2)$, and since $\operatorname{gcd}\left(a_{\chi}, b_{\chi}\right)=1$ we have $b_{\chi} \mid m-2$.

(2) $\left|\chi\left(D_{j}\right)\right|^{2}$ is an algebraic integer, and by (5.4) also takes both rational values $\frac{\left(b_{\chi}+a_{\chi}\right) \lambda}{b_{\chi}-a_{\chi}}$ and $\frac{\left(b_{\chi}-a_{\chi}\right) \lambda}{b_{\chi}+a_{\chi}}$ as $j$ ranges over $\{1,2, \ldots, m\}$. Therefore $\frac{\left(b_{\chi}+a_{\chi}\right) \lambda}{b_{\chi}-a_{\chi}}$ and $\frac{\left(b_{\chi}-a_{\chi}\right) \lambda}{b_{\chi}+a_{\chi}}$ are both integers.

(3) $|\chi(D)|^{2}$ is an algebraic integer, and by (5.4) is also the rational number $\frac{4 a_{\chi}^{2} \lambda}{b_{\chi}^{2}-a_{\chi}^{2}}$. Therefore $\frac{4 a_{\chi}^{2} \lambda}{b_{\chi}^{2}-a_{\chi}^{2}}$ is an integer, which implies $\left(b_{\chi}^{2}-a_{\chi}^{2}\right) \mid 4 \lambda$. If $b_{\chi}+a_{\chi}$ is odd, then $\operatorname{gcd}\left(b_{\chi}-a_{\chi}, b_{\chi}+a_{\chi}\right)=\operatorname{gcd}\left(2 b_{\chi}, b_{\chi}+a_{\chi}\right)=\operatorname{gcd}\left(b_{\chi}, b_{\chi}+a_{\chi}\right)=1$ 
so that from part (2) we obtain $\left(b_{\chi}-a_{\chi}\right) \mid \lambda$ and $\left(b_{\chi}+a_{\chi}\right) \mid \lambda$ and therefore $\left(b_{\chi}^{2}-a_{\chi}^{2}\right) \mid \lambda$.

Using Lemma 5.1, we recover the result of Proposition 1.4 (1) as Corollary 5.2, and obtain new restrictions for $m \in\{5,6\}$ as Corollary 5.3.

Corollary 5.2 A nontrivial ( $v, m, k, \lambda)-S E D F$ does not exist for $m \in\{3,4\}$.

Corollary 5.3 Let $a_{\chi}, b_{\chi}$ be defined as in (5.1).

(1) If there exists a nontrivial $(v, 5, k, \lambda)-S E D F$ in a group $G$, then $\left(a_{\chi}, b_{\chi}\right)=(1,3)$ and $2 \mid \lambda$ for each $\chi \in \widehat{G}^{N}$.

(2) If there exists a nontrivial $(v, 6, k, \lambda)-\operatorname{SEDF}$ in a group $G$, then $\left(a_{\chi}, b_{\chi}\right)=(1,2)$ and $3 \mid \lambda$ for each $\chi \in \widehat{G}^{N}$.

Motivated by Corollary 5.3, we say that a nontrivial ( $v, m, k, \lambda)$-SEDF with $m>2$ for which $\left(a_{\chi}, b_{\chi}\right)$ takes a constant value $(a, b)$ for all $\chi \in \widehat{G}^{N}$ has the simple character value property with respect to $(a, b)$. In the following subsection we obtain restrictions on SEDFs having this property. In particular, for $m=5$ and for $m=6$ we obtain asymptotic nonexistence results for a family of SEDFs, each of which must have this property with respect to a fixed $(a, b)$ by Corollary 5.3 .

\subsection{The simple character value property}

As above, suppose that $\left\{D_{1}, D_{2}, \ldots, D_{m}\right\}$ is a nontrivial $(v, m, k, \lambda)$-SEDF in a group $G$ with $m>2$, and write $D=\bigcup_{i=1}^{m} D_{i}$. Suppose further that $\left\{D_{1}, D_{2}, \ldots, D_{m}\right\}$ has the simple character value property with respect to $(a, b)$. Then by $(5.3)$, we may partition $\widehat{G}^{N}$ (with respect to $D_{1}$ ) into the disjoint union of the sets

$$
\begin{aligned}
& \widehat{G}^{+}=\left\{\chi \in \widehat{G}^{N} \mid \chi\left(D_{1}\right)=\frac{a+b}{2 a} \chi(D)\right\}, \\
& \widehat{G}^{-}=\left\{\chi \in \widehat{G}^{N} \mid \chi\left(D_{1}\right)=\frac{a-b}{2 a} \chi(D)\right\},
\end{aligned}
$$

and from the definition (2.3), $\widehat{G}$ is the disjoint union $\left\{\chi_{0}\right\} \cup \widehat{G}^{0} \cup \widehat{G}^{+} \cup \widehat{G}^{-}$. By (2.2) and (5.4), we then obtain the character values in Table 1.

We now determine the size of the sets $\widehat{G}^{0}, \widehat{G}^{+}, \widehat{G}^{-}$.

Table 1 Character sums for an SEDF with $m>2$, having the simple character value property with respect to $(a, b)$

\begin{tabular}{llll}
\hline$\chi \in \widehat{G}$ & $|\chi(D)|^{2}$ & $\chi\left(D_{1}\right)$ & $\left|\chi\left(D_{1}\right)\right|^{2}$ \\
\hline$\chi=\chi 0$ & $k^{2} m^{2}$ & $k$ & $k^{2}$ \\
$\chi \in \widehat{G}^{0}$ & 0 & & $\lambda$ \\
$\chi \in \widehat{G}^{+}$ & $\frac{4 a^{2} \lambda}{b^{2}-a^{2}}$ & $\frac{a+b}{2 a} \chi(D)$ & $\frac{(b+a) \lambda}{b-a}$ \\
$\chi \in \widehat{G}^{-}$ & $\frac{4 a^{2} \lambda}{b^{2}-a^{2}}$ & $\frac{a-b}{2 a} \chi(D)$ & $\frac{(b-a) \lambda}{b+a}$ \\
\hline
\end{tabular}


Theorem 5.4 Suppose $\left\{D_{1}, D_{2}, \ldots, D_{m}\right\}$ is a nontrivial $(v, m, k, \lambda)$-SEDF in a group $G$ with $m>2$, having the simple character value property with respect to $(a, b)$. Then the sizes of the sets $\widehat{G}^{0}, \widehat{G}^{+}, \widehat{G}^{-}$(defined as in (2.3), (5.5), (5.6) with reference to the sets $D_{1}$ and $\left.D=\bigcup_{i=1}^{m} D_{i}\right)$ are

$$
\begin{aligned}
\left|\widehat{G}^{0}\right| & =(v-1)\left(1-\frac{\left(b^{2}-a^{2}\right)(v-k m) m}{4 a^{2} k(m-1)}\right), \\
\left|\widehat{G}^{+}\right| & =\frac{(v-1)(v-k m)\left(b^{2}-a^{2}\right)((b-a) m+2 a)}{8 a^{2} b k(m-1)}, \\
\mid \widehat{G}^{-} & =\frac{(v-1)(v-k m)\left(b^{2}-a^{2}\right)((b+a) m-2 a)}{8 a^{2} b k(m-1)},
\end{aligned}
$$

and each of $\left|\widehat{G}^{0}\right|,\left|\widehat{G}^{+}\right|,\left|\widehat{G}^{-}\right|$is a nonnegative integer and $\left|\widehat{G}^{+}\right|+\left|\widehat{G}^{-}\right|>0$.

Proof Each of $\left|\widehat{G}^{0}\right|,\left|\widehat{G}^{+}\right|,\left|\widehat{G}^{-}\right|$is a nonnegative integer by definition, and $\left|\widehat{G}^{+}\right|+$ $\left|\widehat{G}^{-}\right|=\left|\widehat{G}^{N}\right|>0$ by Lemma 2.2. Write $D D^{(-1)}=\sum_{g \in G} c_{g} g \in \mathbb{Z}[G]$. From Proposition 2.1,

$$
c_{1}=\frac{1}{v} \sum_{\chi \in \widehat{G}}|\chi(D)|^{2}
$$

The left side $c_{1}=|D|=k m$ is the coefficient of the identity in the expression $D D^{(-1)}$, and the right side can be evaluated using Table 1 to give

$$
k m=\frac{1}{v}\left(k^{2} m^{2}+\left(v-1-\left|\widehat{G}^{0}\right|\right) \frac{4 a^{2} \lambda}{b^{2}-a^{2}}\right) .
$$

Substitute for $\lambda$ from the counting relation (1.3) to obtain the required expression for $\left|\widehat{G}^{0}\right|$.

Similarly, write $D_{1} D_{1}^{(-1)}=\sum_{g \in G} d_{g} g \in \mathbb{Z}[G]$ and use Proposition 2.1 and Table 1 to give

$$
k=\frac{1}{v}\left(k^{2}+\left|\widehat{G}^{0}\right| \lambda+\left|\widehat{G}^{+}\right| \frac{(b+a) \lambda}{b-a}+\left|\widehat{G}^{-}\right| \frac{(b-a) \lambda}{b+a}\right) .
$$

We now obtain the required expressions for $\left|\widehat{G}^{+}\right|$and $\left|\widehat{G}^{-}\right|$using (5.7) and the counting condition $\left|\widehat{G}^{0}\right|+\left|\widehat{G}^{+}\right|+\left|\widehat{G}^{-}\right|=v-1$.

We obtain the following asymptotic nonexistence result from Theorem 5.4.

Theorem 5.5 Let $m, \lambda, a, b$ be fixed positive integers, where $m>2$ and $b>a$ and $\operatorname{gcd}(a, b)=1$. Then for all sufficiently large $k$, there does not exist a nontrivial $(v, m, k, \lambda)-S E D F$ having the simple character value property with respect to $(a, b)$. 
Proof Apply the condition $\left|\widehat{G}^{0}\right| \geq 0$ to (5.7), and rearrange to give the inequality

$$
\frac{v}{k} \leq m+\frac{4 a^{2}(m-1)}{m\left(b^{2}-a^{2}\right)} \text {. }
$$

Since $m$ and $\lambda$ are fixed, the counting relation (1.3) shows that $v$ grows like $k^{2}$ as $k$ increases. Therefore for all sufficiently large $k$, the inequality in $v / k$ does not hold.

As a consequence of Corollary 5.3 and Theorem 5.5, we obtain the following asymptotic nonexistence result for $m \in\{5,6\}$.

Corollary 5.6 Let $\lambda$ be a fixed positive integer. Then for all sufficiently large $k$, there does not exist a nontrivial $(v, 5, k, \lambda)-S E D F$ and there does not exist a nontrivial $(v, 6, k, \lambda)-S E D F$.

We can obtain results similar to Corollary 5.6 for values of $m$ greater than 6. For example, suppose there exists a nontrivial $(v, 7, k, \lambda)$-SEDF. From Lemma 5.1 we find that $\lambda \bmod 12 \in\{0,4,6,8\}$, and that the SEDF has the simple character value property with respect to $(1,5)$ if $\lambda \bmod 12=6$ and with respect to $(3,5)$ if $\lambda \bmod 12 \in\{4,8\}$. Therefore for fixed $\lambda$ for which $\lambda$ mod $12 \neq 0$, for all sufficiently large $k$ there does not exist a nontrivial $(v, 7, k, \lambda)$-SEDF. Likewise, for fixed $\lambda$ for which $\lambda \bmod 10 \neq 0$, for all sufficiently large $k$ there does not exist a nontrivial $(v, 8, k, \lambda)$-SEDF.

We derive further divisibility conditions on the SEDF parameters in Theorem 5.9. We first require two number-theoretic lemmas.

Lemma 5.7 [14, Lemma 2.3] Let $p$ be a prime and let $e$ be a positive integer. Let $\sigma=\sum_{i=0}^{p^{e}-1} c_{i} \zeta_{p^{e}}^{i}$, where each $c_{i} \in \mathbb{Z}$. Then $\sigma=0$ if and only if $c_{i}=c_{j}$ for all $i$ and $j$ satisfying $i \equiv j\left(\bmod p^{e-1}\right)$.

Lemma 5.8 Let $p$ be a prime and $H$ be a p-group. Let $E=\sum_{h \in H} c_{h} h \in \mathbb{Z}[H]$, where each $c_{h} \geq 0$ and $\sum_{h \in H} c_{h}=u$. Suppose there is an integer $\ell$ and a character $\chi \in \widehat{H}$ for which $|\chi(E)|^{2}=\ell$. Then $u^{2}+(p-1) \ell=$ pr for some integer $r \geq u$.

Proof Let $p^{e}=\exp (H)$. Then

$$
\begin{aligned}
\ell & =|\chi(E)|^{2} \\
& =\sum_{h, j \in H} c_{h} c_{j} \chi(h) \overline{\chi(j)} \\
& =\sum_{i=0}^{p^{e}-1} d_{i} \zeta_{p^{e}}^{i},
\end{aligned}
$$

where $d_{i}=\sum_{h, j \in H: \chi(h) \overline{\chi(j)}=\zeta_{p^{e}}^{i}} c_{h} c_{j}$. Each $d_{i}$ is a nonnegative integer, and

$$
\begin{aligned}
\sum_{i=0}^{p^{e}-1} d_{i} & =\sum_{h, j \in H} c_{h} c_{j} \\
& =u^{2} .
\end{aligned}
$$


Subtract $\ell$ from both sides of (5.8), and deduce from Lemma 5.7 that

$$
d_{0}-\ell=d_{p^{e-1}}=d_{2 p^{e-1}}=\cdots=d_{(p-1) p^{e-1}}
$$

and

$$
\begin{array}{r}
d_{j}=d_{p^{e-1}+j}=d_{2 p^{e-1}+j}=\cdots=d_{(p-1) p^{e-1}+j} \\
\text { for each } j \text { satisfying } 1 \leq j \leq p^{e-1}-1 .
\end{array}
$$

Substitute into (5.9) to obtain

$$
p \sum_{i=0}^{p^{e-1}-1} d_{i}-(p-1) \ell=u^{2}
$$

so that $u^{2}+(p-1) \ell=p r$ where $r$ is an integer satisfying

$$
r=\sum_{i=0}^{p^{e-1}-1} d_{i} \geq d_{0}=\sum_{h, j \in H: \chi(h)=\chi(j)} c_{h} c_{j} \geq \sum_{h \in H} c_{h}^{2} \geq \sum_{h \in H} c_{h}=u .
$$

Theorem 5.9 Suppose $\left\{D_{1}, D_{2}, \ldots, D_{m}\right\}$ is a nontrivial $(v, m, k, \lambda)$-SEDF in a group $G$ with $m>2$, having the simple character value property with respect to $(a, b)$, and let $p$ be a prime divisor of $v$. Then either the following both hold:

(1a) $\left|G_{p}\right|$ divides $\mathrm{km}$,

(1b) $k^{2}+\left(\left|G_{p}\right|-1\right) \lambda=\left|G_{p}\right| r_{1}$ for some integer $r_{1} \geq k$.

or the following all hold:

(2a) $k^{2} m^{2}+(p-1) \frac{4 a^{2} \lambda}{b^{2}-a^{2}}=p r_{2}$ for some integer $r_{2} \geq k m$,

(2b) $k^{2}+(p-1) \frac{(b-a) \lambda}{b+a}=$ pr $_{3}$ for some integer $r_{3} \geq k$,

(2c) $k^{2}+(p-1) \frac{(b+a) \lambda}{b-a}=p r_{4}$ for some integer $r_{4}$.

Proof Let $\rho$ be the natural projection from $G$ to $G_{p}$, and let $D=\bigcup_{i=1}^{m} D_{i}$. For each nonprincipal character $\tilde{\chi} \in \widehat{G_{p}}$ and its associated lifting character $\chi \in \widehat{G}$, Table 1 gives

$$
\begin{aligned}
& \left(|\tilde{\chi}(\rho(D))|^{2},\left|\tilde{\chi}\left(\rho\left(D_{1}\right)\right)\right|^{2}\right) \\
& =\left(|\chi(D)|^{2},\left|\chi\left(D_{1}\right)\right|^{2}\right) \\
& = \begin{cases}\left(\frac{4 a^{2} \lambda}{b^{2}-a^{2}}, \frac{(b+a) \lambda}{b-a}\right) \text { or }\left(\frac{4 a^{2} \lambda}{b^{2}-a^{2}}, \frac{(b-a) \lambda}{b+a}\right) & \text { for } \chi \in \widehat{G}^{N}, \\
(0, \lambda) & \text { for } \chi \in \widehat{G}^{0} .\end{cases}
\end{aligned}
$$


Case 1: $\tilde{\chi}(\rho(D))=0$ for every nonprincipal character $\tilde{\chi} \in \widehat{G_{p}}$. Apply Proposition 2.1 with $A=\rho(D)$ to obtain $\rho(D)=\frac{k m}{\left|G_{p}\right|} G_{p}$, giving (1a). By (5.10), we have $\left|\tilde{\chi}\left(\rho\left(D_{1}\right)\right)\right|^{2}=\lambda$ for every nonprincipal character $\tilde{\chi} \in \widehat{G_{p}}$. Apply Proposition 2.1 with $A=\rho\left(D_{1}\right) \rho\left(D_{1}\right)^{(-1)}=\sum_{g \in \widehat{G}_{p}} c_{g} g$ to obtain $c_{1}=\frac{1}{\left|G_{p}\right|}\left(k^{2}+\left(\left|G_{p}\right|-1\right) \lambda\right)$ and note that $c_{1} \geq\left|D_{1}\right|=k$, giving (1b).

Case 2: $\tilde{\chi}(\rho(D)) \neq 0$ for some nonprincipal character $\tilde{\chi} \in \widehat{G_{p}}$. By (5.10), this $\tilde{\chi}$ satisfies $|\tilde{\chi}(\rho(D))|^{2}=\frac{4 a^{2} \lambda}{b^{2}-a^{2}}$, which is an integer by Lemma 5.1 (3). Apply Lemma 5.8 with $(H, E)=\left(G_{p}, \rho(D)\right)$ and $u=|D|=k m$ to give (2a). By (5.10), this $\tilde{\chi}$ also satisfies $\left|\tilde{\chi}\left(\rho\left(D_{1}\right)\right)\right|^{2}=\frac{(b+a) \lambda}{b-a}$ or $\frac{(b-a) \lambda}{b+a}$. Then by (5.4) there is some $j \neq 1$ for which

$$
\left\{\left|\tilde{\chi}\left(\rho\left(D_{1}\right)\right)\right|^{2},\left|\tilde{\chi}\left(\rho\left(D_{j}\right)\right)\right|^{2}\right\}=\left\{\frac{(b+a) \lambda}{b-a}, \frac{(b-a) \lambda}{b+a}\right\},
$$

and both values are integers by Lemma 5.1 (2). Apply Lemma 5.8 with $(H, E)=\left(G_{p}, \rho\left(D_{1}\right)\right)$ and with $(H, E)=\left(G_{p}, \rho\left(D_{j}\right)\right)$ to give $(2 \mathrm{~b})$ and $(2 \mathrm{c})$.

By Proposition 1.4 (2), we know that a nontrivial ( $v, m, k, \lambda)$-SEDF does not exist when $v$ is prime and $m>2$. We now prove a nonexistence result when $v$ is a prime power and $m>2$.

Theorem 5.10 Let $G$ be a group of order $v=p^{s}$ where $p$ is an odd prime, and suppose that 2 is self-conjugate modulo $\exp (G)$. Suppose $\left\{D_{1}, D_{2}, \ldots, D_{m}\right\}$ is a nontrivial $(v, m, k, \lambda)-S E D F$ in $G$ with $m>2$, having the simple character value property with respect to $(a, b)$. Then $a$ and $b$ are both odd.

Proof Suppose, for a contradiction, that $a$ and $b$ are not both odd. Since $\operatorname{gcd}(a, b)=1$, we therefore have $b+a$ odd and so $\left(b^{2}-a^{2}\right) \mid \lambda$ by Lemma 5.1 (3). We shall show that this implies $\mathrm{km}$ and $v-\mathrm{km}$ are both even, contradicting that $v=p^{s}$ for odd $p$.

Write $D=\bigcup_{i=1}^{m} D_{i}$ and use Table 1 to give

$$
|\chi(D)|^{2}=\frac{4 a^{2} \lambda}{b^{2}-a^{2}} \quad \text { for all nonprincipal } \chi \in \widehat{G}^{N} .
$$

Since $\left(b^{2}-a^{2}\right) \mid \lambda$, this gives

$$
\chi(D) \overline{\chi(D)} \equiv 0 \quad\left(\bmod 2^{2}\right) \text { for all nonprincipal } \chi \in \widehat{G}^{N} .
$$

Since 2 is self-conjugate modulo $\exp (G)$, by [5, Chapter VI, Lemma 13.2], we have

$$
\chi(D) \equiv 0 \quad(\bmod 2) \quad \text { for all nonprincipal } \chi \in \widehat{G}^{N} .
$$

By taking a translate of $D$ if necessary, we may assume that $1 \notin D$. Write $D=$ $\sum_{g \in G} d_{g} g \in \mathbb{Z}[G]$. From Proposition 2.1, 


$$
0=v d_{1}=\sum_{\chi \in \widehat{G}} \chi(D)=k m+\sum_{\chi \in \widehat{G}^{N}} \chi(D) .
$$

Combining (5.12) and (5.13), we find that $\mathrm{km}$ is even.

To show that $v-\mathrm{km}$ is even, repeat the above analysis with $D$ replaced by $G \backslash D$, noting that $|\chi(G-D)|^{2}=|\chi(D)|^{2}$ for each nonprincipal $\chi \in \widehat{G}$.

We now illustrate the use of Theorem 5.10 to rule out the existence of an $(81,6,12,9)-$ SEDF and a $(6561,6,984,738)-$ SEDF.

Example 5.11 Suppose, for a contradiction, that there exists an (81, 6, 12, 9)-SEDF or there exists a $(6561,6,984,738)$-SEDF. By Corollary 5.3 (2), these SEDFs have the simple character value property with respect to $(1,2)$. Since 2 is self-conjugate modulo 81 and modulo 6561, Theorem 5.10 then gives the contradiction that 2 is odd.

\subsection{Further nonexistence results}

In this subsection, we extend the analysis of Sect. 5.1 to the case of an SEDF for which the simple character value property does not necessarily hold. Suppose that $\left\{D_{1}, D_{2}, \ldots, D_{m}\right\}$ is a nontrivial $(v, m, k, \lambda)$-SEDF in a group $G$ with $m>2$, and let $D=\bigcup_{i=1}^{m} D_{i}$. Suppose that $\left(a_{\chi}, b_{\chi}\right)$ as defined in (5.1) takes exactly $t \geq 1$ distinct values as $\chi$ ranges over $\widehat{G}^{N}$, so that

$$
\left\{\sqrt{1+\frac{4 \lambda}{|\chi(D)|^{2}}} \mid \chi \in \widehat{G}^{N}\right\}=\left\{\frac{b_{i}}{a_{i}} \mid 1 \leq i \leq t\right\},
$$

where $a_{i}, b_{i} \in \mathbb{Z}$ and $b_{i}>a_{i}>0$ and $\operatorname{gcd}\left(a_{i}, b_{i}\right)=1$. Define

$$
\begin{aligned}
& S^{+}=\left\{\left(\frac{4 a_{i}^{2} \lambda}{b_{i}^{2}-a_{i}^{2}}, \frac{\left(b_{i}+a_{i}\right) \lambda}{b_{i}-a_{i}}\right) \mid 1 \leq i \leq t\right\}, \\
& S^{-}=\left\{\left(\frac{4 a_{i}^{2} \lambda}{b_{i}^{2}-a_{i}^{2}}, \frac{\left(b_{i}-a_{i}\right) \lambda}{b_{i}+a_{i}}\right) \mid 1 \leq i \leq t\right\} .
\end{aligned}
$$

Then from (2.2), (2.3) and (5.4) we have

$$
\begin{cases}\left(|\chi(D)|^{2},\left|\chi\left(D_{1}\right)\right|^{2}\right) \in S^{+} \cup S^{-} & \text {for } \chi \in \widehat{G}^{N}, \\ \left(|\chi(D)|^{2},\left|\chi\left(D_{1}\right)\right|^{2}\right)=(0, \lambda) & \text { for } \chi \in \widehat{G}^{0} .\end{cases}
$$

The following result generalizes Theorem 5.9. The proof, which is omitted, uses (5.14) in a similar manner to the use of (5.10) in the proof of Theorem 5.9.

Theorem 5.12 Suppose $\left\{D_{1}, D_{2}, \ldots, D_{m}\right\}$ is a nontrivial $(v, m, k, \lambda)$-SEDF in a group $G$ with $m>2$, let $p$ be a prime divisor of $v$. Suppose that $\left(a_{\chi}, b_{\chi}\right)$ as defined in (5.1) takes values in the set $\left\{\left(a_{i}, b_{i}\right) \mid 1 \leq i \leq t\right\}$ as $\chi$ ranges over $\widehat{G}^{N}$ (defined with reference to the set $\left.D=\bigcup_{i=1}^{m} D_{i}\right)$. Then either the following both hold: 
(1a) $\left|G_{p}\right|$ divides $\mathrm{km}$,

(1b) $k^{2}+\left(\left|G_{p}\right|-1\right) \lambda=\left|G_{p}\right| r_{1}$ for some integer $r_{1} \geq k$.

or, for some $i$ satisfying $1 \leq i \leq t$, the following all hold:

(2a) $k^{2} m^{2}+(p-1) \frac{4 a_{i}^{2} \lambda}{b_{i}^{2}-a_{i}^{2}}=p r_{2, i}$ for some integer $r_{2, i} \geq k m$,

(2b) $k^{2}+(p-1) \frac{\left(b_{i}-a_{i}\right) \lambda}{b_{i}+a_{i}}=p r_{3, i}$ for some integer $r_{3, i} \geq k$,

(2c) $k^{2}+(p-1) \frac{\left(b_{i}+a_{i}\right) \lambda}{b_{i}-a_{i}}=p r_{4, i}$ for some integer $r_{4, i}$.

We now illustrate the use of Theorem 5.12 to rule out the existence of a $(676,26,18,12)-$ SEDF and a $(2401,37,60,54)-$ SEDF.

Example 5.13 Suppose, for a contradiction, that there exists a $(676,26,18,12)$-SEDF. By Lemma 5.1,

$$
\left(a_{\chi}, b_{\chi}\right) \in\{(1,2),(1,3)\} \text { for all } \chi \in \widehat{G}^{N} \text {. }
$$

For each of these possible values of $\left(a_{\chi}, b_{\chi}\right)$, both (1a) and (2a) of Theorem 5.12 fail with $p=13$, giving the required contradiction.

Example 5.14 Suppose, for a contradiction, that there exists a $(2401,37,60,54)$ SEDF. By Lemma 5.1,

$$
\left(a_{\chi}, b_{\chi}\right) \in\{(1,5),(5,7)\} \text { for all } \chi \in \widehat{G}^{N} \text {. }
$$

We cannot have $\left(a_{\chi}, b_{\chi}\right)=(1,5)$ for $\chi \in \widehat{G}^{N}$, otherwise both (1a) and (2a) of Theorem 5.12 fail with $p=7$. Therefore $\left(a_{\chi}, b_{\chi}\right)=(5,7)$ for all $\chi \in \widehat{G}^{N}$, and so the SEDF satisfies the simple character value property with respect to $(5,7)$. Theorem 5.4 then gives $\left|\widehat{G}^{0}\right|=\frac{9212}{15}$, which contradicts that $\left|\widehat{G}^{0}\right|$ is an integer.

We now extend the nonexistence result of Theorem 5.10 for $v$ a prime power and $m>2$.

Theorem 5.15 Let $v=p^{s}$ for a prime $p$, and let $a_{\chi}, b_{\chi}$ be defined as in (5.1) with reference to the set $D=\bigcup_{i=1}^{m} D_{i}$ associated with a nontrivial $(v, m, k, \lambda)$-SEDF $\left\{D_{1}, D_{2}, \ldots, D_{m}\right\}$ in a group $G$ with $m>2$. Let

$T_{\chi}=\left\{\frac{4 a_{\chi}^{2} \lambda}{b_{\chi}^{2}-a_{\chi}^{2}}, \frac{\left(b_{\chi}+a_{\chi}\right) \lambda}{b_{\chi}-a_{\chi}}, \frac{\left(b_{\chi}-a_{\chi}\right) \lambda}{b_{\chi}+a_{\chi}}\right\}$ and $U_{\chi}= \begin{cases}T_{\chi} & \text { if }\left|\widehat{G}^{0}\right|=0 \\ T_{\chi} \cup\{\lambda\} & \text { if }\left|\widehat{G}^{0}\right|>0\end{cases}$

For each $u \in U_{\chi}$, if $q$ is a prime divisor of $u$ and $q$ is a primitive root modulo $p^{s}$, then $q^{f} \| u$ for some even $f$.

Proof For each $u \in U_{\chi}$, by (2.2), (2.3) and (5.4) there is a subset $E_{\chi}$ of $G$ for which $\left|\chi\left(E_{\chi}\right)\right|^{2}=u$. Since $|G|=p^{s}$, we have $\exp (G)=p^{e}$ for some integer $e \leq s$ and so $\chi\left(E_{\chi}\right) \in \mathbb{Z}\left[\zeta_{p^{e}}\right]$. Now if $q$ is a primitive root modulo $p^{s}$, then $q$ is a primitive root modulo $p^{e}$. Apply Lemma 4.1 with $X=\chi\left(E_{\chi}\right)$. 
We now illustrate the use of Theorem 5.15 to rule out the existence of a $(6561,42,120,90)-$ SEDF.

Example 5.16 Suppose, for a contradiction, that there exists a $(6561,42,120,90)$ SEDF. By Lemma 5.1,

$$
\left(a_{\chi}, b_{\chi}\right) \in\{(1,2),(1,4),(1,5),(4,5)\} \text { for all } \chi \in \widehat{G}^{N}
$$

Since 5 is a primitive root modulo 6561, by Theorem 5.15 we cannot have $\left(a_{\chi}, b_{\chi}\right) \in$ $\{(1,2),(1,5),(4,5)\}$ otherwise the set $T_{\chi}$ defined in (5.15) contains an element $u$ for which $5 \| u$. Therefore $\left(a_{\chi}, b_{\chi}\right)=(1,4)$ for all $\chi \in \widehat{G}^{N}$, and so the SEDF satisfies the simple character value property with respect to $(1,4)$. Since 2 is self-conjugate modulo 6561 , Theorem 5.10 then gives the contradiction that 4 is odd.

Remark 5.17 Combination of the nonexistence results of Proposition 1.4 (4), Lemmas 1.5, 5.1, and Theorems 4.2, 5.4, 5.9, 5.10, 5.12, 5.15, shows that there is no nontrivial $(v, m, k, \lambda)$-SEDF for $v \leq 10^{5}$ and $m \in\{5,6\}$; and that for $v \leq 10^{4}$ and $m>2$ there are only 70 possible parameter sets for a nontrivial $(v, m, k, \lambda)$-SEDF that is not near-complete, namely:

\begin{tabular}{llllllllllll}
\hline$v$ & $m$ & $k$ & $\lambda$ & $v$ & $m$ & $k$ & $\lambda$ & $v$ & $m$ & $k$ & $\lambda$ \\
\hline 540 & 12 & 42 & 36 & 3381 & 23 & 130 & 110 & 5832 & 8 & 714 & 612 \\
784 & 30 & 18 & 12 & 3888 & 24 & 156 & 144 & 5832 & 18 & 147 & 63 \\
1089 & 35 & 24 & 18 & 3888 & 47 & 52 & 32 & 5832 & 18 & 294 & 252 \\
1540 & 77 & 18 & 16 & 3888 & 47 & 78 & 72 & 5832 & 35 & 98 & 56 \\
1701 & 35 & 30 & 18 & 3969 & 32 & 112 & 98 & 5832 & 86 & 49 & 35 \\
1701 & 35 & 40 & 32 & 4096 & 8 & 390 & 260 & 5888 & 92 & 58 & 52 \\
2058 & 86 & 22 & 20 & 4096 & 14 & 105 & 35 & 6400 & 80 & 54 & 36 \\
2376 & 11 & 190 & 152 & 4096 & 14 & 210 & 140 & 6656 & 26 & 121 & 55 \\
2401 & 7 & 280 & 196 & 4225 & 67 & 48 & 36 & 6656 & 26 & 242 & 220 \\
2401 & 9 & 60 & 12 & 4375 & 7 & 162 & 36 & 6860 & 20 & 266 & 196 \\
2401 & 9 & 120 & 48 & 4375 & 7 & 324 & 144 & 6860 & 58 & 95 & 75 \\
2401 & 9 & 180 & 108 & 4375 & 7 & 486 & 324 & 6976 & 218 & 30 & 28 \\
2401 & 9 & 240 & 192 & 4375 & 7 & 540 & 400 & 8281 & 93 & 60 & 40 \\
2401 & 16 & 120 & 90 & 4375 & 9 & 405 & 300 & 8625 & 23 & 140 & 50 \\
2401 & 37 & 40 & 24 & 4375 & 16 & 270 & 250 & 8625 & 23 & 280 & 200 \\
2401 & 65 & 30 & 24 & 4375 & 37 & 108 & 96 & 8960 & 7 & 1054 & 744 \\
2500 & 18 & 105 & 75 & 4375 & 37 & 54 & 24 & 8960 & 32 & 238 & 196 \\
2500 & 35 & 42 & 24 & 4375 & 37 & 81 & 54 & 9801 & 13 & 420 & 216 \\
2500 & 52 & 42 & 36 & 4564 & 163 & 26 & 24 & 9801 & 26 & 308 & 242 \\
2601 & 53 & 40 & 32 & 4625 & 37 & 68 & 36 & 9801 & 57 & 140 & 112 \\
2625 & 42 & 48 & 36 & 5376 & 44 & 75 & 45 & 9801 & 101 & 70 & 50 \\
2646 & 16 & 138 & 108 & 5376 & 44 & 100 & 80 & 9801 & 101 & 84 & 72 \\
2784 & 116 & 22 & 20 & 5776 & 78 & 60 & 48 & & & & \\
3025 & 57 & 36 & 24 & 5832 & 8 & 595 & 425 & & & & \\
\hline & & & & & & & & & & &
\end{tabular}

In Sect. 4 we proved the exponent bound of Theorem 4.2 using only information about the SEDF parameters $(v, m, k, \lambda)$, and applied it to the case $m=2$. We now 
derive a different exponent bound that uses information about the possible values of $|\chi(D)|^{2}$ and $\left|\chi\left(D_{1}\right)\right|^{2}$, and apply it to two of the open cases with $m>2$ given in Remark 5.17.

Theorem 5.18 Suppose $\left\{D_{1}, D_{2}, \ldots, D_{m}\right\}$ is a $(v, m, k, \lambda)-S E D F$ in a group $G$, let $D=\bigcup_{i=1}^{m} D_{i}$, and let $p$ be a prime dividing $v$. Suppose $U$ is a subgroup of $G$ for which $U \cap G_{p}=\{1\}$ and $p$ is self-conjugate modulo $\exp (G / U)$.

(1) If $|\chi(D)|^{2} \equiv 0\left(\bmod p^{2 d}\right)$ for every nonprincipal $\chi \in \widehat{G}$, then $\exp \left(G_{p}\right) \leq$ $\max \left\{\frac{|U|}{p^{d}}\left|G_{p}\right|, \frac{p\left|\widehat{G}^{0}\right| \cdot|U|}{(p-1) v}\left|G_{p}\right|\right\}$.

(2) If $\left|\chi\left(D_{1}\right)\right|^{2} \equiv 0\left(\bmod p^{2 d}\right)$ for every nonprincipal $\chi \in \widehat{G}$, then $\exp \left(G_{p}\right) \leq$ $\frac{|U|}{p^{d}}\left|G_{p}\right|$.

Proof The proof is analogous to that of [5, Chapter VI, Theorem 15.11]. We prove only (1); the proof of (2) is similar.

Let $W$ be a subgroup of $G_{p}$ for which $G_{p} / W$ is cyclic of order $\exp \left(G_{p}\right)$. It follows from $U \cap G_{p}=\{1\}$ that $U \cap W=\{1\}$, and so we may write $H=U \times W$. Since $\exp \left(G_{p} / W\right)=\exp \left(G_{p}\right)$, we then have $\exp (G / H)=\exp (G / U)$. Let $\rho$ be the canonical epimorphism $\rho: G \rightarrow G / H$. Then by assumption,

$$
|\widetilde{\chi}(\rho(D))|^{2} \equiv 0 \quad\left(\bmod p^{2 d}\right) \text { for every nonprincipal } \tilde{\chi} \in \widehat{G / H}
$$

Since $p$ is self-conjugate modulo $\exp (G / H)$, this implies [5, Chapter VI, Lemma 13.2]

$$
\tilde{\chi}(\rho(D)) \equiv 0 \quad\left(\bmod p^{d}\right) \text { for every nonprincipal } \tilde{\chi} \in \widehat{G / H}
$$

and then by Ma's Lemma [19] we have

$$
\rho(D)=p^{d} X_{0}+P X_{1}
$$

where $X_{0}, X_{1} \in \mathbb{Z}[G / H]$ have nonnegative coefficients and $P$ is the unique subgroup of $G / H$ of order $p$.

In the case $X_{0} \neq 0$, we have $p^{d} \leq|H|=|U| \cdot|W|=|U| \cdot \frac{\left|G_{p}\right|}{\exp \left(G_{p}\right)}$, which rearranges to $\exp \left(G_{p}\right) \leq \frac{|U|}{p^{d}}\left|G_{p}\right|$.

Otherwise, in the case $X_{0}=0$, we have $\rho(D)=P X_{1}$. Now consider the $\frac{|G|}{|H|}\left(1-\frac{1}{p}\right)$ characters $\tilde{\chi} \in \widehat{G / H}$ which are nonprincipal on $P$. Each such character satisfies $\tilde{\chi}(\rho(D))=0$, and its associated lifting character $\chi \in \widehat{G}$ satisfies $\chi(D)=0$. Therefore by the definition (2.3) of $\widehat{G}^{0}$, we have $\left|\widehat{G}^{0}\right| \geq \frac{|G|}{|H|}\left(1-\frac{1}{p}\right)$, which implies $\exp \left(G_{p}\right) \leq$ $\frac{p\left|\widehat{G}^{0}\right| \cdot|U|}{(p-1) v}\left|G_{p}\right|$.

We now illustrate the use of Theorem 5.18 to obtain an exponent bound on a group containing a $(2401,7,280,196)-$ SEDF and a group containing a $(5832,8,595,425)$ SEDF. 
Example 5.19 Suppose there exists a $(2401,7,280,196)$-SEDF in a group $G$. Note that $196=2^{2} \cdot 7^{2}$ and that neither 2 nor 7 is a primitive root modulo $7^{4}=2401$, so Theorem 4.2 does not apply. However, by Lemma 5.1 the SEDF satisfies the simple character value property with respect to $(a, b)=(3,5)$, and so from Table 1 we have $\left|\chi\left(D_{1}\right)\right|^{2} \in\left\{7^{2}, 4 \cdot 7^{2}, 16 \cdot 7^{2}\right\}$ for every nonprincipal $\chi \in \widehat{G}$. Since 7 is self-conjugate modulo 2401, we may apply Theorem $5.18(2)$ with $(p, d)=(7,1)$ and $U=\{1\}$ to show that $\exp (G) \leq 7^{3}$.

Example 5.20 Suppose there exists a $(5832,8,595,425)-S E D F$ in $G$. Note that $5832=2^{3} \cdot 3^{6}$ and $425=5^{2} \cdot 17$. Theorem 4.2 does not give any constraint on the structure of $G$ (even though it may be applied with $(p, q)=(3,5))$. By Lemma 5.1, the SEDF satisfies the simple character value property with respect to $(2,3)$, and so from Table 1 we have $|\chi(D)|^{2} \in\left\{0,2^{4} \cdot 5 \cdot 17\right\}$ and $\left|\chi\left(D_{1}\right)\right|^{2} \in\left\{5 \cdot 17,5^{2} \cdot 17,5^{3} \cdot 17\right\}$ for every nonprincipal $\chi \in \widehat{G}$, and $\left|\widehat{G}^{0}\right|=2079$ from Theorem 5.4. In this case Theorem 5.18 (2) does not apply. However, because 2 is self-conjugate modulo $2^{3} \cdot 3^{6}$, we may apply Theorem $5.18(1)$ with $(p, d)=(2,2)$ and $U=\{1\}$ to obtain $\exp \left(G_{2}\right) \leq \max \left\{2, \frac{154}{27}\right\}<2^{3}$. Therefore $\exp \left(G_{2}\right) \leq 2^{2}$.

\section{Concluding remarks}

We have presented a comprehensive treatment of SEDFs, using character theory and algebraic number theory to derive many nonexistence results. We have characterized the parameters of a nontrivial near-complete SEDF, and constructed a $(243,11,22,20)$-SEDF in $\mathbb{Z}_{3}^{5}$ from a detailed analysis of the action of the Mathieu group $M_{11}$ on the points of the projective geometry $P G(4,3)$. This is the first known nontrivial example of SEDF with $m>2$.

As we were finalizing our paper, Wen, Yang and Feng posted a preprint [27] in which they independently constructed a $(243,11,22,20)$-SEDF in $\mathbb{Z}_{3}^{5}$ using cyclotomic classes over $\mathbb{F}_{35}$. Their method, which was also used to construct some generalizations of SEDFs [28], is very different from ours.

In closing, we note that until now SEDFs have been considered only in abelian groups. We ask: are there examples of nontrivial SEDFs in nonabelian groups?

Acknowledgements We are grateful to Ruizhong Wei for kindly supplying a preprint of the paper [3]. We thank the referee for providing very careful and helpful comments.

\section{References}

1. Abbott, R., Bray, J., Linton, S., Nickerson, S., Norton, S., Parker, R., Suleiman, I., Tripp, J., Walsh, P., Wilson., R.: ATLAS of finite group representations-version 3. http://brauer.maths.qmul.ac.uk/Atlas/ $\mathrm{v} 3 /$ matrep/M11G1-f3r5aB0

2. Arasu, K.T., Jungnickel, D., Ma, S.L., Pott, A.: Strongly regular Cayley graphs with $\lambda-\mu=-1$. J. Combin. Theory Ser. A 67(1), 116-125 (1994)

3. Bao, J., Ji, L., Wei, R., Zhang, Y.: New existence and nonexistence results for strong external difference families (2016) arXiv:1612.08385v2

4. Berlekamp, E.R., van Lint, J.H., Seidel, J.J.: A strongly regular graph derived from the perfect ternary Golay code. In: A Survey of Combinatorial Theory (Proc. Internat. Sympos., Colorado State Univ., Fort Collins, Colo., 1971), pp. 25-30. North-Holland, Amsterdam (1973) 
5. Beth, T., Jungnickel, D., Lenz, H.: Design Theory. Vol. I, Volume 69 of Encyclopedia of Mathematics and its Applications, 2nd edn. Cambridge University Press, Cambridge (1999)

6. Calderbank, R., Kantor, W.M.: The geometry of two-weight codes. Bull. Lond. Math. Soc. 18(2), 97-122 (1986)

7. Chang, Y., Ding, C.: Constructions of external difference families and disjoint difference families. Des. Codes Cryptogr. 40(2), 167-185 (2006)

8. Cramer, R., Dodis, Y., Fehr, S., Padró, C., Wichs, D.: Detection of algebraic manipulation with applications to robust secret sharing and fuzzy extractors. In: Advances in Cryptology-EUROCRYPT 2008. Lecture Notes in Comput. Sci., vol. 4965, pp. 471-488. Springer, Berlin (2008)

9. Cramer, R., Fehr, S., Padró, C.: Algebraic manipulation detection codes. Sci. China Math. 56(7), 1349-1358 (2013)

10. Cramer, R., Padró, C., Xing, C.: Optimal algebraic manipulation detection codes in the constant-error model. In: Theory of Cryptography. Part I, Lecture Notes in Comput. Sci., vol. 9014, pp. 481-501. Springer, Heidelberg (2015)

11. Davis, J.A., Huczynska, S., Mullen, G.L.: Near-complete external difference families. Des. Codes Cryptogr. 84(3), 415-424 (2017)

12. Ding, C.: Two constructions of $(v,(v-1) / 2,(v-3) / 2)$ difference families. J. Combin. Des. 16(2), 164-171 (2008)

13. Dixon, J.D., Mortimer, B.: Permutation Groups. Graduate Texts in Mathematics, vol. 163. Springer, New York (1996)

14. Hiramine, Y.: On abelian $(2 n, n, 2 n, 2)$-difference sets. J. Combin. Theory Ser. A 117(7), 996-1003 (2010)

15. Huczynska, S., Paterson, M.B.: Existence and non-existence results for strong external difference families. Discrete Math. 341(1), 87-95 (2018)

16. Ireland, K., Rosen, M.: A Classical Introduction to Modern Number Theory. Graduate Texts in Mathematics, vol. 84, 2nd edn. Springer, New York (1990)

17. Leung, K.H., Ma, S.L.: Partial difference sets with Paley parameters. Bull. Lond. Math. Soc. 27(6), 553-564 (1995)

18. Levenshtein, V.I.: Combinatorial problems motivated by comma-free codes. J. Combin. Des. 12(3), 184-196 (2004)

19. Ma, S.L.: Polynomial addition sets. PhD Thesis, University of Hong Kong (1985)

20. Ma, S.L.: A survey of partial difference sets. Des. Codes Cryptogr. 4(3), 221-261 (1994)

21. Martin, W.J., Stinson, D.R.: Some nonexistence results for strong external difference families using character theory. Bull. ICA 80, 79-92 (2017)

22. Mihăilescu, P.: Primary cyclotomic units and a proof of Catalan's conjecture. J. Reine Angew. Math. 572, 167-195 (2004)

23. Ogata, W., Kurosawa, K., Stinson, D.R., Saido, H.: New combinatorial designs and their applications to authentication codes and secret sharing schemes. Discrete Math. 279(1-3), 383-405 (2004)

24. Paley, R.E.A.C.: On orthogonal matrices. J. Math. Phys. 12, 311-320 (1933)

25. Paterson, M.B., Stinson, D.R.: Combinatorial characterizations of algebraic manipulation detection codes involving generalized difference families. Discrete Math. 339(12), 2891-2906 (2016)

26. Polhill, J.: Paley type partial difference sets in non p-groups. Des. Codes Cryptogr. 52(2), 163-169 (2009)

27. Wen, J., Yang, M., Feng, K.: The $(n, m, k, \lambda)$-strong external difference family with $m \geq 5$ exists (2016). arXiv:1612.09495

28. Wen, J., Yang, M., Fu, F., Feng, K.: Cyclotomic construction of strong external difference families in finite fields. Des. Codes Cryptogr. (2017). https://doi.org/10.1007/s10623-017-0384-y 